\title{
The Globular Cluster System in the Outer Regions of NGC 4472
}

\author{
Katherine L. Rhode ${ }^{1,2}$ and Stephen E. Zepf ${ }^{1}$ \\ Department of Astronomy, Yale University, New Haven, CT 06520; \\ rhode@astro.yale.edu, zepf@astro.yale.edu
}

\begin{abstract}
We have undertaken a study of the globular cluster (GC) systems of a large sample of elliptical and spiral galaxies in order to test specific predictions of galaxy formation models. Here we present results for the first elliptical in the study, the giant Virgo cluster galaxy NGC 4472 (M49). The galaxy was observed in three filters (BVR) using the wide-field Mosaic Imager on the Kitt Peak 4-meter telescope. The Mosaic images roughly double the radial coverage of previous CCD observations of NGC 4472. We have combined the Mosaic data with published spectroscopic data and archival HST observations in order to study NGC 4472's GC system in detail, and to fully characterize the amount of contamination in our sample of GC candidates. We find that the radial profile of the GC system is fit fairly well by a de Vaucouleurs law of the form $\log \sigma_{G C}=(3.38 \pm 0.08)-(1.56 \pm 0.05) r^{1 / 4}$ out to $17^{\prime}(\sim 80 \mathrm{kpc})$, but that the observed profile falls slightly below the de Vaucouleurs law between $17^{\prime}$ and $23^{\prime}$, the limit of the data. The bimodal color distribution observed in previous studies is apparent in our data. We find a small metallicity gradient in the inner $8^{\prime}$ of the total GC system due to the increasing ratio of blue to red clusters, consistent with results from past studies. The gradient vanishes, however, when the entire radial extent of the data is taken into account. We estimate a total of $~ 5,900$ GCs in NGC 4472 out to $23^{\prime}$, yielding a specific frequency of $3.6 \pm 0.6$ within this radius. This specific frequency value is smaller than that found by previous studies of NGC 4472. We examine the implications of these results with regard to predictions made by four different galaxy formation models, and find that all four models have at least one inconsistency with the data.
\end{abstract}

Subject headings: galaxies: formation; galaxies: star clusters; galaxies: individual (NGC 4472)

\footnotetext{
${ }^{1}$ Visiting Astronomer, Kitt Peak National Observatory, National Optical Astronomy Observatories, which is operated by the Association of Universities for Research in Astronomy, Inc. (AURA) under cooperative agreement with the National Science Foundation

${ }^{2}$ NASA Graduate Student Researchers Program Fellow
} 


\section{Introduction}

Globular clusters (GCs) are among the oldest readily-observable objects in the Universe, and have been detected in all types of massive galaxies and many dwarfs. The fact that GCs have such large ages and are seen in virtually all types of galaxies indicates that they are closely tied to galaxy formation. Studying the ensemble properties of the entire system of GCs around a given galaxy can provide important clues regarding the formation and subsequent evolution of that galaxy, and such studies can be particularly useful for testing theories of elliptical galaxy formation. GC systems of ellipticals are typically more populous than those of spirals (cf. Ashman \& Zepf 1998). Moreover, GC systems are generally at least as extended as the light distributions of their host galaxies (cf. Harris 1986, Ashman \& Zepf 1998), so GCs can be used to probe the formation and enrichment history of the outer regions of elliptical galaxies.

Recent studies have found that the GC systems of many ellipticals have bimodal rather than single-peaked color distributions (e.g., Geisler et al. 1996, Kundu \& Whitmore 1999, Gebhardt \& Kissler-Patig 1999). The presence of two peaks has been interpreted as due to two GC populations with different metallicities, possibly formed in different episodes. Evidence also suggests that at least in some ellipticals, the bluer (metal-poor) GC population is more extended than the red (metal-rich) population (e.g., Geisler et al. 1996, Neilsen 2000). These observations agree in general with the predictions of a galaxy formation scenario proposed by Ashman \& Zepf (1992), in which an elliptical galaxy is formed via the merger of two or more disk galaxies. In the Ashman \& Zepf picture, a younger, spatially-concentrated, metal-rich system of GCs is formed during the merger itself, and the spatially-extended, metal-poor GC population originates in the halos of the progenitor spirals. Other models may also explain the observed differences between the GC populations in ellipticals. Forbes et al. (1997) suggest that the GCs in ellipticals are formed in distinct episodes, with the metal-poor population formed early, from less enriched gas, and the metal-rich population forming later, along with the galaxy field stars. In the case of giant ellipticals and $\mathrm{cD}$ galaxies, Forbes et al. suggest that tidal stripping of GCs from nearby galaxies may contribute to building up the outer GC system. Another model, proposed by Côté et al. (1998), suggests that giant ellipticals form via dissipational collapse, producing a metal-rich GC population. Metal-poor GCs are then captured from other galaxies by accretion or tidal stripping.

These scenarios make different predictions for the detailed properties of the GC systems of ellipticals. For example, the merger scenario of Ashman \& Zepf predicts that the mass-normalized number of metal-poor GCs around ellipticals and spirals should be the same, since in this model all of the metal-poor GCs come from the progenitor spirals. The Côté et al. model predicts that a significant number of metal-poor, tidally-stripped GCs should reside at large distances from giant cluster ellipticals. We have undertaken a study of a large sample of elliptical and spiral galaxies in order to test the predictions of the various formation models. Using a new generation of wide-field CCD imagers, we have observed a sample of early-type galaxies in the Virgo cluster and the field, as well as a large sample of fairly isolated, edge-on spirals. Our aim is to accurately quantify the total numbers, spatial distributions and color distributions of the GC systems in these galaxies out 
to large radii. By observing the galaxies in three filters and employing image classification analysis we can greatly reduce the amount of potential contamination (from background and foreground objects) in our GC samples, while simultaneously detecting GCs further out than previous CCD studies have done. Furthermore, we make use of available space-based and spectroscopic data to help quantify the amount of contamination that exists in our GC lists.

Here we present the results of our study of the GC system of the galaxy NGC 4472 (M49). NGC 4472 is located four degrees from the center of the Virgo cluster and is the brightest of the Virgo galaxies. We chose to analyze NGC 4472's GC system first because the abundance of Hubble Space Telescope (HST) and spectroscopic data currently available made it possible for us to fully quantify the amount of contamination in our data. Studying this galaxy has allowed us to test our overall methods and ensure that they are sufficient for accomplishing the goals of the project. In the following section, the observations and initial data reduction are described. Section 3 describes subsequent analysis steps performed on the data, and Section 1 gives the results. Section 月 consists of a discussion of the implications of the results with regard to various galaxy formation models, and the final section contains a summary of the paper.

\section{Observations and Reductions}

Observations of NGC 4472 were obtained in March 1999 with the Mosaic Imager on the 4-meter Mayall telescope at Kitt Peak National Observatory (KPNO). The Mosaic Imager consists of eight 2048 x 4096 SITe CCDs. The CCDs are separated by small ( 50 pixels) gaps. When mounted on the 4-meter, each pixel of the Mosaic subtends $0.26^{\prime \prime}$ on the sky, resulting in a total field-of-view $36^{\prime}$ on a side. We imaged the galaxy in three broadband filters $(B V R)$. Five exposures were taken through each filter, and the telescope was dithered in a predetermined pattern on the sky, in order to help eliminate cosmic rays as well as blank regions in the images due to the gaps between the CCDs. Total integration times were $3300 \mathrm{~s}$ in $B, 2700 \mathrm{~s}$ in $V$, and $2100 \mathrm{~s}$ in $R$. The $V$ and $R$ images were taken under fairly clear, although not photometric, conditions, but the $B$ image was taken under poorer sky conditions. Observations in three filters were taken of three more early-type galaxies (NGC 3379, NGC 4406, and NGC 4594) during the same observing run; results for these objects will be presented in a subsequent paper (Rhode \& Zepf 2001).

Data reduction was accomplished using the IRAF package MSCRED, which is designed especially for the reduction of images from mosaic CCD detectors. We reduced the data using standard practices, i.e., ZEROCOMBINE to create a combined bias image, FLATCOMBINE and SFLATCOMBINE to create combined dome flats and twilight sky flats, MEDIAN to smooth the sky flats, and CCDPROC to perform overscan level subtraction, bias image subtraction,

\footnotetext{
${ }^{3}$ IRAF is distributed by the National Optical Astronomy Observatories, which are operated by the Association of Universities for Research in Astronomy, Inc., under cooperative agreement with the National Science Foundation.
} 
and flat-field division on the object images. We then used the MSCRED tasks MSCZERO, MSCCMATCH and MSCIMAGE to construct single images from the multi-extension mosaic images, by mapping the pixels from each FITS extension to a single grid on the sky. A constant sky background was subtracted from the resultant individual images using the task IMSURFIT. Finally, multiple images in a given filter were scaled to a common flux level and combined into a single "stacked" image using pixel rejection to eliminate cosmic rays. A constant sky level, measured from the image used as a reference image during the scaling step, was added to each of the stacked images to restore the background counts. The resolution (point-spread function FWHM) of the final stacked images is $1.1^{\prime \prime}$ in $B, 1.1^{\prime \prime}$ in $V$, and $1.3^{\prime \prime}$ in $R$.

\section{Data Analysis}

\subsection{Detection of the GC System}

The primary observational signature of a GC system around a distant galaxy is an overdensity of compact objects around the galaxy with magnitudes and colors in the expected range for GCs. At a distance of $\sim 16 \mathrm{Mpc}$, GCs around NGC 4472 are unresolved in our images. To detect the GCs, we began by smoothing each of the three stacked images using a circular filter with a diameter 6 times the average FWHM in the image. The light from the galaxy was then removed by subtracting this smoothed image from the original. A constant was added to the resultant images to restore the background level, and the $B V R$ images were aligned to each other. Next the IRAF task DAOFIND was used to search for GC candidates in the images. A few small regions of the images - e.g., around saturated stars, or on the edges of the frames, where the noise level was higher - were excluded from the search because DAOFIND tended to find spurious sources in those regions. A list of 4,329 GC candidates was produced by requiring that the candidates be detected in all three filters.

\section{2. $\quad$ Extended Source Cut}

To eliminate contaminating objects (specifically, background galaxies) from the list of GC candidates, the FWHM values of the light profiles of the 4,329 sources were measured and plotted versus their instrumental magnitudes. Objects with FWHM values that were much larger than the average for the image were presumed to be extended objects and were removed from the GC candidate list.

Figure 1 shows the FWHM versus magnitude values for the 4,329 GC candidates in the $V$ and $R$ images. Filled circles mark the accepted objects, and open squares are objects thought to be extended and therefore eliminated from the GC list. The figure shows that at bright magnitudes, point sources tend to have FWHM values that scatter around a mean value and form a distinct 
sequence in the FWHM versus magnitude plane. As the signal-to-noise ratio decreases at fainter magnitudes, however, the sequence spreads out and it becomes more difficult to distinguish between point sources and extended objects. We took this into account by accepting a larger range of FWHM values for sources with fainter magnitudes. Sources that had FWHM values too small to be believable were also removed from the GC candidate list in this process. By checking the results of our extended source cut against HST WFPC2 images of NGC 4472 (see Section 3.6.1), we found that the best results were obtained by basing the extended source cut on the $V$ and $R$ images only, and excluding the $B$ image (which was taken under deteriorating sky conditions) from this process. 871 sources that appeared extended in either the $V$ or $R$ image were eliminated in the extended source cut, leaving 3,458 objects in the GC candidate list.

\subsection{Photometry and Photometric Calibration}

Because the GC candidates are faint, the most accurate photometry can be achieved by measuring their light in a small aperture and applying an aperture correction to account for the light missing from the part of the profile that has been excluded. We determined an aperture correction for each of the three stacked images by measuring $\sim 20-30$ bright, unsaturated stars in each image and measuring their magnitudes in a series of apertures from 1-6 times the average FWHM in the image. The measured aperture corrections and associated errors are given in Table 11. The errors range from 0.01 to 0.02 magnitudes, and reflect the fact that the point spread function varies slightly across a given Mosaic frame. Using a single aperture correction for a given stacked image limits the accuracy of the final photometry to 0.01 to 0.02 magnitudes.

The 4-m observations of NGC 4472 were taken during non-photometric conditions, so in order to calibrate them, we observed the galaxy with the WIYN 3.5-m telescope at KPNO on a photometric night in January 2000. One $600 \mathrm{~s}$ exposure of NGC 4472 was taken through each filter $(B V R)$. Calibration frames were taken throughout the night. A total of nine standard stars (Landolt 1992) were used to calibrate the WIYN images. Twenty-three bright, but not saturated, stars were found in common between the WIYN and 4-m images and used to "bootstrap" the 4-m calibration to the WIYN calibration. The WIYN images were essentially treated as standard star fields; magnitudes and colors of the 23 bright stars were measured in both images, and photometric coefficients (zero points and color terms) were derived to calibrate the 4-m data to the WIYN data.

Finally, aperture photometry was carried out in all three filters on the 3,458 GC candidates in the Mosaic frames, using an aperture of radius equal to the average FWHM of each image. Calibrated total magnitudes for the sources were calculated using the aperture corrections listed in

\footnotetext{
${ }^{4}$ The WIYN Observatory is a joint facility of the University of Wisconsin-Madison, Indiana University, Yale University, and the National Optical Astronomy Observatories.
} 
Table 1 and the photometric coefficients derived via the bootstrapping method. The magnitudes were corrected for Galactic extinction using reddening maps from Schlegel et al. (1998), which give values of $A_{B}=0.087, A_{V}=0.067$, and $A_{R}=0.057$ in the direction of NGC 4472 .

To check the accuracy of our photometric calibration, we compared the $R$ magnitudes of 20 of our GC candidates with magnitudes from a study of NGC 4472's GC system published by Geisler et al. (1996). Because Geisler et al. did not publish equatorial positions for their GCs, we used a list of objects published in Sharples et al. (1998), who obtained spectra for 55 of the GC candidates from the Geisler et al. study. The Sharples et al. paper tabulates precise coordinates for the sources, and lists the $T_{1}$ and $C-T_{1}$ values from Geisler et al. We used the coordinates of the objects to find matches between their list of GC candidates and ours, then used a transformation given in Geisler (1996) to convert the $T_{1}$ magnitudes to $R$. The mean difference between our $R$ magnitudes and those calculated from the Geisler et al. data is 0.005, with a standard deviation of 0.035. Geisler et al. estimate their photometric errors to be $\sim 0.03$ magnitudes, and ours are comparable. This indicates excellent agreement between our photometry and that from Geisler et al.

\subsection{Color Selection}

The fact that we observe our target galaxies with three broadband filters allows us to significantly reduce the amount of contamination in our GC samples, because we can then use two colors to differentiate the GCs from other stellar populations or background objects. Before beginning this project, we investigated which color combinations would help us to adequately isolate the GCs in the color-color plane. We found that by using $B V R$ filters, we could distinguish GCs from the majority of other types of objects, while not having to use impractically long exposure times. Figure $2 a$ shows where GCs would be expected to lie in the BVR color-color plane. The large filled circles are Milky Way GCs from the Harris catalog (Harris 1996). We used the Harris catalog to derive a linear relationship between $B-V$ and $V-R$ color for Milky Way GCs. This relationship serves as the mid-line of the rectangular box shown. The mid-line extends from $B-V=0.56$ to 0.99 , which corresponds to an $[\mathrm{Fe} / \mathrm{H}]$ range of -2.5 to 0.0 for Galactic GCs. The rectangle is drawn by extending the fitted $V-R$ versus $B-V$ relationship by \pm 2 times the RMS scatter about the fit. The positions of galaxies of different morphological types are shown in the figure as "tracks" the galaxies would follow with increasing redshift. The tracks were made using template spectra from Kennicutt (1992), Coleman, Wu \& Weedman (1980), and Kinney et al. (1996). The spectra were redshifted from $\mathrm{z}=0$ to $\mathrm{z}=0.7$ in steps of 0.025 , and then sampled through the $B V R$ filter transmission curves to arrive at the data plotted in the figure.

Figure $2 a$ shows that GCs should occupy a fairly narrow region in the $B V R$ color-color plane, and also that the galaxies that have colors similar to GCs are, for the most part, late-type objects with low to moderate redshifts. Many of these background galaxies can be eliminated with image classification analysis, but others would be impossible to eliminate using only ground-based 
photometric data. For this reason we have used space-based and spectroscopic data to help us quantify the amount of contamination in the sample from such objects (see Section 3.6).

To eliminate non-GCs from our list of 3,458 GC candidates, we devised a selection algorithm that works as follows: (1) If a GC candidate has a V magnitude brighter than 19.5 (the brightest magnitude for GCs in the Virgo elliptical M87 (Whitmore et al. 1995)), it is removed from the list. (2) If the GC candidate has $B-V$ and $V-R$ colors that place it within the rectangular region shown in Figure $2 a$, or within a distance from the rectangle equal to its formal photometric error, it is retained in the sample. A total of 1,465 objects pass the magnitude and color criteria imposed on the GC candidate list; these objects serve as the final sample of GC candidates. The positions of the accepted objects in the $B V R$ color-color plane are shown in Figure $2 b$. Because the photometric errors for these objects were taken into account in the color selection step, they occupy a region broader than the rectangular area shown in Figure $2 a$.

\subsection{Completeness Tests}

To investigate the GC detection limit in our images, we ran a series of tests of the completeness in each filter. For these tests, 800 artificial point sources of known magnitude were added to each image, covering a range of 5-7 magnitudes at 0.1-magnitude intervals. The same detection steps used on the original image (i.e., DAOFIND with a given detection threshold) were applied to the images containing the artificial sources, to see what fraction of the objects in each magnitude interval would be detected. The results from these tests yield the completeness as a function of magnitude for each filter; these completeness functions are shown in Figure 3. The 50\% completeness limits in the three filters are: $B=23.33, V=23.65$, and $R=22.83$.

The $B$ image was taken under poor sky conditions and is therefore much more shallow than intended. This has serious consequences for the completeness of our GC sample as a whole. Because typical $B-V$ colors for GCs in ellipticals lie in the range $0.55-1.0$, the $B$ image must be that much deeper than the $V$ image in order for all of the GCs that are detected in $V$ to be detected in $B$ as well. Our $B$ image is in fact slightly more shallow than the $V$ image. The implications of this are that in our overall sample of GC candidates, we are better able to detect blue GCs (since redder GCs will not show up as well in our relatively shallow $B$ image), and also that the comparatively poor completeness of the $B$ image will dominate the completeness corrections we make in later steps (e.g., in the correction for magnitude incompleteness made in the calculation of the total number of GCs; see Sections 3.7 and 4.3).

\subsection{Contamination}

For ground-based, photometric studies of extragalactic GC systems, one of the most serious potential problems is contamination of the GC candidate sample by foreground and background 
objects. By selecting GC candidates using image analysis and color selection in three filters, contamination can in principle be greatly reduced, although not eliminated completely. We used space-based and ground-based data, as well as theoretical models, to quantify the amount of contamination existing in our GC sample.

\subsubsection{HST Data}

The Hubble Space Telescope has the advantage of being able to resolve many faint compact background galaxies that might appear as point sources in ground-based images and could contaminate a GC sample. To make use of this fact, we analyzed all of the publicly-available Wide-Field and Planetary Camera 2 (WFPC2) images of NGC 4472 taken with the F555W filter, the filter that most closely resembles the $V$ filter. There were four data sets that fit these criteria: HST program GO.5236, PI: Westphal; HST program GO.6673, PI: Baum; HST program GO.5920, PI: Brodie; and HST program GO.6352, PI: Ferguson. We requested that "On-The-Fly" calibration be applied to the images returned by the HST data archive.

Images of a given pointing that had been dithered were shifted so that they were aligned to each other. The CRREJ task, in the STSDAS package, was then used to create combined, cosmic-ray-rejected images of each pointing in the four data sets. A few of the pointings slightly overlapped each other. We eliminated three small sections of images from the rest of the analysis in order to avoid duplicating the coverage of any area on the sky. Excluding the redundant regions, the total area around NGC 4472 covered by the HST images is 22.05 square arc minutes. The IRAF task WCSCTRAN was used to translate the sky positions of the 1,465 GC candidates into pixel positions in the WFPC2 images. A total of 133 of these sources were located in the WFPC2 images, distributed over two planetary camera (PC) fields and 12 Wide Field camera (WF) fields.

We followed the method used by Kundu et al. (1999) to distinguish between point sources (bona fide GC candidates) and extended objects (contaminating background galaxies) in the WFPC2 images. Aperture photometry was performed on the 133 sources using aperture radii of 0.5 pixels and 3 pixels and a sky annulus from 5 to 8 pixels. Kundu et al. found that the ratio of fluxes in the two apertures is a good criterion for eliminating background galaxies: objects appearing on the PC chip with Counts $_{3 p i x} /$ Counts $_{0.5 p i x}<12$, and on the WF chips with Counts $_{3 \text { pix }} /$ Counts $_{0.5 p i x}<8$, are point sources and thus possible GCs. Using these criteria, we found that five of the $133 \mathrm{GC}$ candidates in the WFPC2 images were actually extended objects.

Besides investigating how many of the GC candidates were likely background galaxies, we also wanted to make use of HST's ability to distinguish between point-like and extended objects in

\footnotetext{
${ }^{5}$ Based on observations made with the NASA/ESA Hubble Space Telescope, obtained from the data archive at the Space Telescope Science Institute. STScI is operated by the Association of Universities for Research in Astronomy, Inc. under NASA contract NAS 5-26555.
} 
order to fine-tune the extended source cut we had applied early in the GC detection process. We found that by using all three of the Mosaic images $(B, V$, and $R)$ to apply the extended source cut, we eliminated some objects that, according to the HST data, were point sources. These objects appeared slightly "blurred out" in the $B$ image because of its relatively poor quality, but were not extended in the $V$ and $R$ Mosaic images. The best results (i.e., most consistent with the results from the HST data) were obtained when we used only the $V$ and $R$ images to apply the extended source cut.

Even with our fine-tuned extended source cut, at faint magnitudes we are still throwing out a few objects that are not intrinsically extended but may appear so because their profiles are just above the noise level of the image. We investigated the profiles of a sample of sources that were excluded in the extended source cut but had colors in the range for GCs. We found that approximately $3.6 \%$ of the GC candidates with the correct colors are mistakenly eliminated in the extended source cut. Both this effect and the contamination of the GC sample are minimized if the sample is cut at $V \leq 23.5$, indicating the importance of signal-to-noise for reliable image classification. Applying this magnitude limit to the GC candidate list leaves a total of 1,461 objects. For this sample, there are four contaminating objects out of a total of 131 GC candidates appearing in the HST images. The contamination can be characterized in terms of a surface density of contaminating objects for the sample of 1,461 GC candidates; there are 4 contaminating objects found in the 22.05 square arc minutes covered by the WFPC2 data, or 0.181 objects per square arc minute. Note that this is an estimate of the amount of contamination existing in our sample from background galaxies, and does not include contamination from foreground stars. The test applied to the HST data delineates between extended objects and point sources only, and it is possible that some of the point sources are Galactic stars. Therefore this is a lower limit on the contamination in the sample.

\subsubsection{Spectroscopic Data}

Another way to distinguish GCs around a galaxy from background or foreground objects is to measure their radial velocities with spectroscopy. Sharples et al. (1998) used a wide-field imaging spectrograph on the 4.2-m Herschel Telescope to obtain radial velocities for 55 GC candidates around NGC 4472. 47 of these were confirmed as GCs, 6 had velocities consistent with their being Galactic stars, and 2 were likely background galaxies. Zepf et al. (2000) used a multi-object spectrograph on the Canada-France-Hawaii Telescope to obtain 120 radial velocities for GC candidates around NGC 4472, finding that 100 of these were GCs, 7 were foreground objects, and 13 were background objects.

We took the combined results from the Sharples et al. (1998) and Zepf et al. (2000) studies and compared them to our GC candidate list to confirm that our estimate of the amount of contamination in the GC sample based on the HST data was correct. The combined spectroscopic data set (excluding duplicates) contained 134 GCs and 28 contaminating objects. We found that 
16 of the 28 contaminating objects ( 5 stars and 11 galaxies) were present in our sample of GC candidates.

In order to make a meaningful comparison between the spectroscopic data and our GC sample, we binned the spectroscopic object list into radial bins from $1^{\prime}$ to $9^{\prime}$ (as far out as the data extended). For each radial bin, we calculated the contamination fraction, and the total number of contaminating objects (stars and galaxies) found in that bin. We executed the same steps for our GC sample data: we binned the data and calculated a local contamination fraction, based on the surface density of contaminating objects from the HST data ( 0.181 objects per square arc minute), and the area contained in the radial bin region. We used this contamination fraction to calculate how many contaminating objects we would have expected to find in the spectroscopic sample of 150 objects. Note that this expected number of contaminating objects includes only galaxies, since the HST data could not distinguish between GCs and stars.

Eleven galaxies from the spectroscopic sample were included in our sample of 1,461 GC candidates. The expected number of galaxies based on the estimate from the HST data is 9. This number is consistent, within Poisson errors, with the actual number found. Therefore, results from two independent methods used to estimate the contamination in our sample due to background galaxies are consistent with each other.

The fact that five of the 16 contaminating objects from the spectroscopic sample found in our GC sample were stars indicates that to account for foreground objects, we should increase the number density estimate of contaminating objects by $\sim 50 \%$ (or, more precisely, by the ratio of 5 stars to 11 galaxies). This increases the estimated surface density of contaminating objects from 0.181 per square arc minute to 0.264 per square arc minute.

We wished to check that this estimate of the surface density of foreground stars was reasonable. Accordingly, the Bahcall-Soneira model of the Galactic halo (Bahcall \& Soneira 1980) was used to provide an estimate of the surface density of foreground objects that might appear in our GC sample. Since the GC sample contains a limited magnitude and color range of objects, we imposed a similar selection on the star counts output from the model. A selection using two colors $(B-V$ and $V-R)$ was not easily implemented in the model code, so we used a $B-V$ cut only. The model predicts that the number density of stars in the direction of NGC 4472 with $V$ magnitude between 19.5 and 23.5, and $B-V$ color between 0.55 and 1.0, is 114 stars per square degree. Our estimate of the number density of objects in the GC sample, based on the spectroscopic data, is 297 stars per square degree, 2.6 times larger than the model prediction. For this reason we conclude that we are very likely overestimating the amount of stellar contamination that exists in our GC sample. 


\subsubsection{Estimate of the Overall Contamination in the GC Sample}

Using two independent methods (HST data and ground-based spectroscopic data) to estimate the amount of contamination in our GC sample due to background galaxies, we find that there are approximately 0.181 galaxies per square arc minute in our sample. We also used two methods (the spectroscopic data, and Bahcall-Soneira model of the Galaxy) to estimate the contamination from

foreground stars, and conclude that an upper limit for the number density of foreground objects in our sample is 0.083 stars per square arc minute. Thus the total surface density of contaminating objects in our sample is $\lesssim 0.264$ objects per square arc minute. We make use of this estimate in calculations of the radial distribution and total number of GCs around NGC 4472, described in Section 4 .

\subsection{Globular Cluster Luminosity Function}

In order to calculate the total number of GCs around NGC 4472, it is necessary to quantify what fraction of the total GC population (as characterized by the intrinsic Globular Cluster Luminosity Function (GCLF) for the galaxy) was detected given the magnitude limits of the images. Corrections for magnitude incompleteness in all three filters, and for contamination from foreground and background objects, must first be applied to the observed GCLF. Then the corrected GCLF can be fit to a GCLF representing the intrinsic one for the galaxy, and the fraction of area covered by the observed GCLF calculated. The intrinsic GCLF is traditionally parameterized by a Gaussian function of a given peak magnitude and dispersion.

A number of studies of NGC 4472's GCLF have been published in recent years. For example, Lee et al. (1998) used ground-based data to determine the GCLF in the Washington $T_{1}$ band. Converting their result to $V$ and applying the value of $A_{V}$ obtained from the Schlegel et al. (1998) maps yields a GCLF peak at $m_{V}^{0}=23.77 \pm 0.10$ with dispersion $\sigma=1.3$. Their GCLF determination came with a number of caveats; for example, it was affected by contamination from background galaxies at the faint end of their observed luminosity function. Puzia et al. (1999) used archival HST data to investigate whether the GCLF turnover varies in different regions of the galaxy, and in the individual (red and blue) GC populations. For the total population, they find the GCLF turnover at $m_{V}^{0}=23.76 \pm 0.20$. Lee \& Kim (2000) used the same HST data to study the GCLF and color distribution of GCs within $4^{\prime}$ of the center of NGC 4472. They found

$m_{V}^{0}=23.50 \pm 0.16$ and $\sigma=1.19 \pm 0.09$. The HST observations used for Puzia et al. and Lee \& Kim reached $\sim 1$ magnitude past the GCLF peak. By far the best-determined GCLF for an elliptical galaxy in Virgo is that published by Whitmore et al. (1995) using HST observations of M87. They observed 1,032 GCs in M87 down to a limiting magnitude of $V=26$, more than 2 magnitudes beyond the GCLF turnover. Because their data reached so far past the peak, their determination of both the location of the peak and, especially, the dispersion of the GCLF are the most robust of these studies. Whitmore et al. found $m_{V}^{0}=23.72 \pm 0.06$ and $\sigma=1.40 \pm 0.06$. We adopt this as the 
best estimate of NGC 4472's intrinsic GCLF. Note that the Whitmore et al. GCLF is consistent within the errors with all the others mentioned, with the exception that the Lee \& Kim (2000) dispersion, $1.19 \pm 0.09$, is significantly more narrow. We attempted to fit our observed GCLF to the Lee \& Kim (2000) GCLF but found that our data are more consistent with a GCLF with a larger dispersion.

We derived an observed GCLF by binning the $V$ magnitudes of 1,279 GC candidates with $V$ $\leq 23.5$ and radial distance $<16^{\prime}$ into 0.4-magnitude-wide bins. Both the magnitude limit and the radial cut were imposed to minimize the amount of contamination in the sample. (The number density of GCs falls off with increasing radius, but the number density of contaminating objects is roughly constant; therefore the fraction of contaminating objects in the sample increases with distance from the galaxy center.) The number of GCs in each bin was multiplied by a correction factor to account for the fractional contamination present in the sample.

To correct the observed GCLF for magnitude incompleteness, our completeness curves (see Section 3.5), were used to calculate the completeness in each filter for each $V$ magnitude bin. The completeness in the $V$ filter was calculated by simply interpolating from the $V$ completeness curve. Calculating the completeness for the other two filters was more complicated. The $B$ completeness that should be applied to a given $V$ bin varies because the $B-V$ colors of the GCs in the $V$ bin vary over the $B-V$ range of the sample. For each of five $B-V$ colors $(0.55$ to 0.95 , at 0.1-magnitude intervals), the $B$ magnitude corresponding to the given $V$ bin was calculated (i.e., $B=(B-V)+V)$. The completeness for each of the five $B$ magnitudes was interpolated from the $B$ completeness curve. The average of these five completeness values was adopted as the $B$ completeness for that particular $V$ bin. This process was repeated for all $V$ bins. A similar series of steps was performed to calculate the $R$ completeness for each $V$ bin; a range of five colors was again used ( $V-R=0.34$ to 0.6 , in steps of 0.065 magnitude) to find the average $R$ completeness corresponding to a given $V$ bin. The completenesses in the three filters were then convolved to calculate a total fractional completeness for each $V$ magnitude bin. The number of GCs in each bin was divided by this total completeness to produce the corrected GCLF. The observed and corrected GCLF's are shown in Figure 4 ; the solid line marks the observed GCLF and the dashed line the corrected function. The constraint that the GC candidates be detected in all three filters, coupled with the lack of depth of the $B$ image, means that the observed $V$ GCLF of the GC candidates covers less of the intrinsic GCLF than desired (see Section 3.5.

Next, a least-squares fit was performed in order to fit the corrected GCLF to the GCLF from Whitmore et al. (1995). Bins with completeness $<50 \%$ were excluded from the fit. The normalization of the Whitmore et al. GCLF was varied so as to minimize $\chi^{2}$. The fractional area of the normalized Whitmore et al. GCLF covered by our observed (uncorrected) GCLF was calculated. This value, 0.236 , gives the fraction of GCs we detected from the total population of the GC system of NGC 4472. 


\section{Results}

\subsection{Radial Distribution}

We used the sample of $1,461 \mathrm{GC}$ candidates with $V \leq 23.5$ to investigate the radial distribution of GCs around NGC 4472. First, the GC candidates were assigned to $1^{\prime}$-wide bins from $1^{\prime}$ to $23^{\prime}$ according to their projected radial distances from the center of the galaxy. An effective area was calculated for each annular region. The effective area equals the area of the annulus, minus the area of regions excluded from the DAOFIND search for GCs, and the area of any part of the annulus that extends past the edge of the Mosaic image. The correction to effective area is very small for those annuli with central radius $\leq 17^{\prime}$.

To correct for contamination, a subtractive correction (equal to the number density of contaminating objects times the effective area of the annulus) was applied to the number of GCs in each radial bin. A small multiplicative correction (amounting to multiplying the total number by 1.04) was then applied to the number of GCs, to account for faint point sources missing from the

GC list because they were incorrectly excluded in the extended source cut (see Section 3.6.1). The total number of GCs in each bin was corrected for incomplete coverage of the GCLF, calculated in Section 3.7. Finally, the surface density of GCs and its associated error (calculated from the Poisson error of the number of GCs, and taking into account errors on the contamination correction and the correction for GC candidates mistakenly excluded during the extended source cut) was calculated for each bin, to produce the radial profile shown in Figure 5 and tabulated in Table 2. The table lists the central radius of each annular region, the number density of GCs within that region, and the fraction of the annulus for which we have data.

Radial profiles of elliptical galaxy GC systems have traditionally been fit with power laws or de Vaucouleurs law profiles, either of which has provided an adequate fit over the previously-observed radial ranges, except for the inner $1-2 \mathrm{kpc}$. Fitting a single power law to the radial profile yields: $\log \sigma_{G C}=(1.91 \pm 0.03)-(1.28 \pm 0.04) \log r$, where $\sigma_{G C}$ is the surface density of GCs and $r$ is in arc minutes. The single power law does not provide a good fit over the entire radial range, however; the surface density falls well below the power law at large radius. A de Vaucouleurs profile, shown as a dotted line in the bottom panel of Figure 5, provides a somewhat better fit: $\log \sigma_{G C}=(3.38 \pm 0.08)-(1.56 \pm 0.05) r^{1 / 4}$. The de Vaucouleurs fit also seems to slightly overestimate the surface density at large radius, but in this case at least the best-fit line intercepts nearly all of the error bars in the profile.

The surface density of GCs in the corrected profile is consistent with zero in the last three bins $\left(21^{\prime}\right.$ to $\left.23^{\prime}\right)$, and is negative in the $23^{\prime}$ bin. These outer bins have the largest correction for missing area, since portions of the outer annuli extend beyond the boundaries of the Mosaic images. Thus it is not clear whether we have actually seen the entire extent of the GC system. On the other hand, the data do indicate that the distribution of clusters does not continue as a power law to radii beyond $23^{\prime}$ and the observed data fall slightly below the de Vaucouleurs profile (which 
falls off more rapidly than a power law) at those large radii. So while we may not have seen a clear "edge" to the GC system, we do seem to have seen the point at which the GC system begins to taper off. Still, additional data - with good spatial coverage beyond $23^{\prime}$ - must be obtained in order for this result to be confirmed.

\subsection{Color Distribution}

As discussed in Section 3.5, the B-band Mosaic image from our data set has a $50 \%$ completeness limit that is slightly brighter than the $V$ image, which means it is much more shallow than intended. As a result we are more sensitive to detecting blue GCs than red ones. The effect that the shallowness of the $B$ image has on the GC sample is apparent in Figure 6, which shows the color-magnitude diagram for the 1,465 GC candidates. The detection limit for these objects becomes brighter as $B-R$ gets larger (i.e., as the objects become redder). In order to say something about the color distribution of the GCs at large radius around NGC 4472, we needed to define a sub-sample from our GC list that was equally complete in red and blue clusters. To do so, we noted that the reddest object in the total sample had $B-R=1.9$. By requiring that all clusters with $B-R \leq 1.9$ must be detected, one can cut the sample at a given $B$ completeness level (e.g., $50 \%$ ) and use that $B$ magnitude and $B-R$ color to define at what $R$ magnitude the sample must be cut. For example, the GC sample is $90 \%$ complete at $B=22.75$, so to include the entire red GC population, the GC candidate list must be cut at $R=20.85$. Doing this yields a sample of 366 sources that we will refer to as the $90 \%$ sample; their color distribution is plotted in Figure 17. The distribution is clearly bimodal, with peaks at $B-R \sim 1.10$ and 1.35, and a gap at $B-R=1.23$. If we simply split the color distribution at the location of the apparent gap, we find that there are 146 and 220 GCs in the red and blue samples, respectively. This yields a ratio of red to blue GCs of $N_{R G C} / N_{B G C}=0.66$.

To quantify the level of bimodality in the observed color distribution, we applied the KMM algorithm (Ashman et al. 1994) to the 90\% sample. KMM tests whether a user-specified number of Gaussian functions provides a better fit to a given distribution than a single Gaussian, and evaluates the improvement in the fit for the multi-component model compared to the singlecomponent model. We find that for the $90 \%$ sample, the hypothesis that the color distribution is drawn from a unimodal parent population is rejected with greater than $99.99 \%$ confidence. KMM also locates the peaks of the subpopulations and provides an estimate of the relative proportion of objects in each subpopulation. KMM can be run using either a constant dispersion for the Gaussian functions, or allowing the dispersions to vary freely (although the second option must admittedly be used with some caution).

The ratio of the red to blue subpopulations in the $90 \%$ sample scatters around the value we estimated above using a simple split of the populations at the location of the gap. KMM yields a lower ratio if a fixed dispersion is used, and a higher one if the dispersions are allowed to vary. Based on the results from our "simple" split and the KMM test, we estimate the ratio of red to 
blue clusters to be approximately 0.7 , although we note that this number may be uncertain by up to 0.2 . In any case, the ratio $N_{R G C} / N_{B G C}$ is likely to be less than one; the implications of this result will be discussed in Section 5, when we compare our results with the predictions of galaxy formation models.

There are too few GC candidates in the $90 \%$ sample to produce robust radial profiles for the red and blue GC populations individually. Instead we took the total list of 1,465 GC candidates and separated it at the location of the gap in the $90 \%$ sample, $B-R=1.23$. Radial profiles were generated for each sample and fit with power laws; the slope for the red sample was $-1.34 \pm 0.08$, and for the blue sample, $-1.26 \pm 0.06$. So although the power law fit for the red clusters is slightly more steep, the difference is not significant.

An alternative method for comparing the radial distributions of the two populations is to simply plot the colors of the GC candidates versus their radial distances. This plot, made using the $90 \%$ sample, is shown in Figure 8. Lee et al. (1998) studied the GC system of NGC 4472 to a radius of $7^{\prime}(\sim 32 \mathrm{kpc})$ and found a strong radial gradient in the color distribution of the total GC population, in the sense that the GC system becomes bluer with increasing radius. They found little, if any, gradient in the individual blue and red populations, and so concluded that the gradient in the total population is due to the varying radial mixture of the two populations - the red clusters are more centrally concentrated than the blue clusters. Figure 8 shows an apparent overdensity of blue clusters in the region between $\sim 4^{\prime}$ and $8^{\prime}$, which could give rise to the same type of color gradient. When we fit a line to the inner portion $\left(<8^{\prime}\right)$ of the $B-R$ versus radius plot, we find a slightly negative slope: $\Delta(B-R) / \Delta r=-0.010 \pm 0.007$. Thus we find similarly to Lee et al. (1998) that the GC system as a whole gets slightly bluer with increasing radius — due to the increasing ratio of blue to red clusters - in this inner region.

Beyond $\sim 8^{\prime}$, however, the overdensity of blue GCs disappears and the overall color distribution becomes more uniform. The gradient caused by the changing ratio of blue clusters to red clusters appears to flatten out as a result. This is borne out by a fit to the color distribution over the entire radial range of the data, to $22^{\prime}$. The slope for the total population over the whole radial range is consistent with zero: $\Delta(B-R) / \Delta r=0.000 \pm 0.002$.

It is possible that the observed lack of a gradient is caused by the increasing fraction of contaminating objects at larger radii. Because there are fewer GCs as the distance from the galaxy center increases, but the number density of contaminating objects is roughly constant, the fraction of contaminating objects in the sample increases with radius. If for some reason the contaminating objects are preferentially red, then a larger fraction of them in the outer regions could cause the measured overall gradient to be zero.

In order to translate our observed color gradients to metallicity gradients, we used the Harris catalog of Galactic GCs (Harris 1996) to derive a relationship between $B-R$ and $[\mathrm{Fe} / \mathrm{H}]$. We used this relationship to convert $B-R$ to $[\mathrm{Fe} / \mathrm{H}]$ and then fit lines to $[\mathrm{Fe} / \mathrm{H}]$ versus the $\log$ of the projected radial distance for GCs in the inner, outer, and full radial range. The 
gradients $(\Delta[\mathrm{Fe} / \mathrm{H}] / \Delta \log (r))$ are $-0.08 \pm 0.09$ for the full range, $-0.27 \pm 0.17$ for the inner $8^{\prime}$, and $+0.76 \pm 0.34$ for the region from $8^{\prime}$ to $23^{\prime}$. Our metallicity gradient for the inner radial range falls between that found by Puzia et al. (1999), who surveyed the inner $4.2^{\prime}$ of the GC system of NGC 4472 and obtained $\Delta[\mathrm{Fe} / \mathrm{H}] / \Delta \log (r)=-0.15 \pm 0.02$, and the Geisler et al. (1996) gradient, which was $-0.41 \pm 0.03$.

\subsection{Specific Frequency}

The number of globular clusters in a galaxy can be characterized by the specific frequency $\left(S_{N}\right)$, the number of GCs normalized by the galaxy luminosity. Specific frequency is defined as:

$$
S_{N} \equiv N_{G C} 10^{+0.4\left(M_{V}+15\right)}
$$

(Harris \& van den Bergh 1981). It is instructive to calculate both the global $S_{N}$ value for a given galaxy, and to investigate the local specific frequency, or how $S_{N}$ varies with radius within the galaxy.

\subsubsection{Local Specific Frequency}

Calculating the local specific frequency requires knowledge of the radial variation of the galaxy light, so that the number of GCs at each radius can be normalized by the galaxy luminosity at that same location. The light distribution of NGC 4472 has been measured in a number of previous studies. For example, King (1978) used photographic data to measure the galaxy's surface brightness out to a radius of $\sim 17^{\prime}$. Fitting a de Vaucouleurs law to King's data yields $\mu_{V}=(13.76 \pm 0.14)+(2.52 \pm 0.05) r^{1 / 4}$. Caon et al. (1994) combined data from CCD images and photographic plates to measure NGC 4472's light distribution out to $r \sim 22^{\prime}$. The values for the effective radius $\left(r_{e}\right)$ and corresponding effective surface brightness $\left(\mu_{e}\right)$ from Caon et al., when converted from $B$ to $V$ using a mean $B-V$ value for NGC 4472 of 0.95 , yield a de Vaucouleurs law of the form $\mu_{V}=14.80+2.15 r^{1 / 4}$. Most recently, Kim et al. (2000) used CCD images to perform surface photometry of NGC 4472 in the Washington $C$ and $T_{1}$ bands, with radial coverage to $\sim 9^{\prime}$. They found that the surface brightness distribution in the outer regions of NGC 4472 (beyond $r=7^{\prime \prime}$ ) can be fit approximately with a de Vaucouleurs law of the form $\mu_{V}=13.62+$ $(2.52 \pm 0.07) r^{1 / 4}$. (Here the $T_{1}$ intercept given in their paper has been converted to a $V$-band value, using the conversion given in Geisler (1996).)

To measure the galaxy light from our data, we used the IRAF task ELLIPSE (in the STSDAS package) to perform surface photometry on the $V$-band Mosaic image of NGC 4472. We used a bad pixel mask to exclude regions of the image containing bright stars and other objects that would interfere with the galaxy photometry. We measured the sky background using a median algorithm in an annulur region with inner radius $16.5^{\prime}$ and outer radius $17.5^{\prime}$, and subtracted 
the measured sky level from the image. The galaxy light was then measured in elliptical regions with semi-major axes from $47^{\prime \prime}$ to $620^{\prime \prime}$. Fitting a de Vaucouleurs profile to the resultant surface brightness profile yields $\mu_{V}=(14.23 \pm 0.08)+(2.30 \pm 0.02) r^{1 / 4}$. Here, $r$ is equal to $\sqrt{a b}$, the mean of the semi-major and semi-minor axes. We adopt this as the galaxy profile for the $S_{N}$ versus radius calculation. Note that our values for the de Vaucouleurs law coefficients fall between the values found by Caon et al. (1994) and Kim et al. (2000).

Figure 9 shows the variation of the specific frequency with radius for NGC 4472. The data are given in Table 3. The filled triangles in the figure are local $S_{N}$ values calculated using our GC data and surface brightness profile for NGC 4472. Error bars include Poisson errors on the number of GCs at each radius, and errors on the corrections we applied to account for contamination and objects mistakenly excluded during the extended-source cut (see Section 4.1). The solid line marks the expected rise of $S_{N}$ if one uses the de Vaucouleurs law fit to the radial distribution of GCs to calculate $S_{N}$ at each radius. Values of $S_{N}$ from the data rise approximately linearly with radius out to $\sim 10^{\prime}$. Beyond that point, the values scatter substantially above and below the solid line out to $\sim 16^{\prime}$. At larger radii, all of the data points fall below the line, similarly to the way they do in the radial profile shown in Figure 5 .

Lee et al. (1998) studied NGC 4472's GC system using ground-based $C$ and $T_{1}$ images and investigated the run of $S_{N}$ in NGC 4472 out to $\sim 7^{\prime}$. Their values for local $S_{N}$ are substantially larger than we find for that radial range. This is due in part to the fact that we are using a smaller distance modulus (31.12, versus their 31.2) and a more shallow galaxy surface brightness profile, both of which result in a more luminous galaxy (and therefore a smaller specific frequency) at each radius. However, even when we use their galaxy profile (published in Kim et al. (2000)) and their distance modulus to calculate $S_{N}$ from our GC distribution, we calculate $S_{N}$ values anywhere from $\sim 20$ to 80 percent smaller than their published values.

There were significant differences in the way the GC sample was selected in Lee et al. (1998) compared to the steps we used. They used a single color $\left(C-T_{1}\right)$ to select GCs from the sample of point sources they detected around NGC 4472, and were not able to apply image classification analysis to their sample to eliminate extended sources. They estimated the amount of contamination in their sample by taking a number density of contaminating objects around NGC 4472 from a photographic study by Harris (1986) and estimating what fraction of those objects would be included after their $C-T_{1}$ cut. For our study, GCs were selected using image classification and two-color photometry, and archival HST data and published spectroscopic observations were used to accurately quantify the amount of contamination. Consequently a possible explanation for the discrepancy between our $S_{N}$ values and theirs is that their sample contained a larger fraction of contaminating objects, resulting in larger $S_{N}$ values at each radius.

To investigate this possibility, we created a sample of GC candidates from our data by taking the original sample of 4,329 sources found in all three $(B V R)$ Mosaic images and applying a single color cut to the sample. We used a $B-V$ cut, which is admittedly less effective for selecting GCs 
than the $C-T_{1}$ cut used by Lee et al. (1998). As in the Lee et al. study, we did not apply an extended source cut. The result was a list of 2,720 GC candidates. Using this list of sources, we executed the steps necessary to correct the total number of clusters for incomplete coverage of the GCLF (i.e., fitting our observed GCLF to the Whitmore et al. (1995) GCLF and calculating the total area under the Whitmore et al. GCLF curve covered by our data). To correct for contamination, we doubled the number density of contaminating objects estimated for our data in Section 3.6.3. Finally, we calculated local $S_{N}$ versus radius, using the same galaxy profile and distance modulus used by Lee et al. The result closely matches theirs; our points overlap the points from Lee et al. within the error bars. Therefore by using less stringent selection criteria to select our GC sample and underestimating the contamination level, we are able to reproduce the higher local $S_{N}$ values found by Lee et al. We believe that our GC sample is much better constrained than the sample used by Lee et al., and that our local specific frequency values are consequently more accurate.

We investigated the accuracy of our local $S_{N}$ values further using a GC sample identified by Puzia et al. (1999) from archival HST images of NGC 4472. The data from Puzia et al. (1999) are presumably less contaminated than ground-based data because HST's high spatial resolution makes it easier to remove background objects from the GC sample. These data should therefore provide a useful test of whether our local $S_{N}$ values are uniformly too low. We used a list of 705 GCs provided by T. Puzia and the information given in Puzia et al. (1999) to create a radial profile, correct it for incompleteness, and fit it with a de Vaucouleurs law. The best fit was $\log \sigma_{G C}$ $=(3.03 \pm 0.10)-(1.37 \pm 0.09) r^{1 / 4}$. We then calculated local $S_{N}$ versus radius for this radial profile and the best-fit galaxy surface brightness profile from our Mosaic data. The resultant $S_{N}$ values are slightly lower than a few of our values, but match most of them within the error bars. We interpret this as further evidence that our local $S_{N}$ values are not underestimated.

Our findings concerning the local specific frequency of NGC 4472 are relevant to results published in a recent paper by McLaughlin (1999). A long-standing observational result is that GC systems of ellipticals are often more spatially extended than the halo light of the galaxy. This implies that, for some reason, bound clusters were sometimes more likely to form at large galactic radius, from gas with comparatively low densities and pressures. McLaughlin explores this issue for NGC 4472 using a composite GC radial profile, created by combining published CCD and photographic observations of the galaxy's GC system. Using the composite profile and the galaxy profile from Caon et al. (1994), McLaughlin finds that the ratio of the mass surface density of the GC system to that of the stellar component of the galaxy is constant at radii beyond $\sim 15 \mathrm{kpc}$. This in turn implies that the local specific frequency, which is directly proportional to the ratio of the mass density of GCs to stars, is roughly constant throughout NGC 4472's outer halo. We find, on the contrary, that NGC 4472's GC system is substantially more extended than the stellar component; the effective radius of the globular cluster profile $\left(r_{e}\right)$ is several times that of the galaxy light. As a result the local specific frequency, rather than being constant at large radius, increases linearly out to at least $\sim 10^{\prime}(\sim 49 \mathrm{kpc})$, and perhaps somewhat beyond that. 


\subsubsection{Global Specific Frequency}

To calculate the global $S_{N}$ for the GC system of NGC 4472, we begin by calculating the total number of GCs in the galaxy. We can calculate a total number, corrected for magnitude incompleteness, lack of spatial coverage, and contamination from non-GCs, by taking the best-fit de Vaucouleurs law to our radial profile (calculated as described in Section 4.1, and shown in Figure 5) and integrating it from $r=0$ to some outer radius. The choice of outer radius is not obvious. To calculate a total number of GCs for the entire system, one could integrate the de Vaucouleurs profile to $r=\infty$; doing so gives $\sim 11,100$ GCs. Integrating the galaxy profile obtained from our Mosaic data from $r=0$ to $\infty$ yields a total $V$ magnitude for NGC 4472 of $V^{T}=$ 8.01. (Note that this is $\sim 0.4$ magnitudes brighter than the $V$ magnitude given in de Vaucouleurs et al. (1991).) This corresponds to an absolute magnitude of $M_{V}^{T}=-23.11$ for the distance modulus determined by Whitmore et al. (1995), $m-M=31.12$. Using these values yields a specific frequency $S_{N}=6.4$.

The calculation above, however, almost certainly overestimates the total number of GCs. We argue that based on the appearance of the radial profile in Figure 5, integrating to infinity is not a valid choice, since the data points in the profile fall below the de Vaucouleurs fit at large radii, and the surface density of GCs is consistent with zero in the last three bins. A better choice would be to integrate the de Vaucouleurs profile to the limit of the data $\left(23^{\prime}\right.$, or $\left.\sim 110 \mathrm{kpc}\right)$ and calculate an $S_{N}$ value for NGC 4472 out to that radius. Integrating the de Vaucouleurs profile and the galaxy profile to $23^{\prime}$ yields 5,870 clusters and $M_{V}^{T}=-23.05$ for the galaxy. This results in a specific frequency $S_{N}=3.6$.

To calculate an error on the global specific frequency, we need to quantify the amount of error associated with each of the major sources of uncertainty in $S_{N}$. One contribution is the uncertainty in the total number of GCs. Assuming Poisson statistics, we can calculate uncertainties in both the total number of GCs observed, and the number of contaminating objects, and add these in quadrature. Doing this, we find that the uncertainty on the total number of GCs leads to an uncertainty in $S_{N}$ of $\sim 0.1$. A second source of error comes from the uncertainty in the galaxy profile and thus the amount of galaxy light out to $23^{\prime}$. As discussed in Section 4.3.1, other groups have found steeper light profiles for NGC 4472, which leads to a slightly fainter total magnitude at $23^{\prime}$. For example, if we use the galaxy profile from Kim et al. (2000) to calculate $S_{N}$, the galaxy magnitude $M_{V}^{T}$ is -22.91 at $23^{\prime}$, resulting in an $S_{N}$ value of 4.0. (Applying the Caon et al. (1994) profile, on the other hand, produces no change in $S_{N}$.) We estimate that the uncertainty in $S_{N}$ due to the galaxy profile is $\sim 0.5$. Finally, there is some error associated with the correction made to account for our incomplete coverage of the GCLF. The correction varied by $\sim 10 \%$ depending on which observed GCLF was used for the fit to the intrinsic GCLF (i.e., exactly how the observed GCLF was corrected for contamination, and whether a radial cut was applied to the GC sample). This translates to an uncertainty in $S_{N}$ of $\sim 0.4$. Combining the three main sources of uncertainty in quadrature yields a final value of $S_{N}$ for NGC 4472 of $3.6 \pm 0.6$. 
Our value of $S_{N}$ is smaller than values found by authors of previous studies of NGC 4472, who typically found $S_{N}$ values in the range $\sim 5-6$. Studies based on CCD data had much less spatial coverage than our data, usually extending out to only a few to several arc minutes. In such cases, authors extrapolated their observed radial profiles out to some radius. For example, Lee et al. (1998) extrapolated their GC profile out to only $10^{\prime}$, and calculated a specific frequency $S_{N}=4.7 \pm 0.6$ (for $V^{T}=8.4$ and $(m-M)=31.2$ ). Photographic studies had spatial coverage comparable to ours but had higher levels (often more than an order of magnitude higher) of contamination. A photographic study of NGC 4472's GC system by Harris $(1986,1991)$ finds $S_{N}$ $=5.0 \pm 1.4$ (for $V^{T}=8.4,(m-M)=31.3$, and integrating to $\left.25^{\prime}\right)$. The $S_{N}$ values from Lee et al. (1998) and Harris $(1986,1991)$ are both larger than ours, but are consistent within the errors.

We consider the value of $S_{N}=6.4$ - calculated by integrating our profile from $r=0$ to infinity - an absolute upper limit on the total $S_{N}$ for NGC 4472. Previous studies that found $S_{N}$ values of 5-6 likely had more contamination from foreground and background objects than was accounted for. We suggest that $S_{N}=3.6 \pm 0.6$, calculated by integrating our profile to $r=23^{\prime}$, is a more accurate estimate of the true value for NGC 4472, since our observed radial profile indicates that it is unlikely that another $\sim 5,000$ GCs exist at radii beyond $23^{\prime}$.

\section{Comparison to Model Predictions}

Although we have just begun this project and therefore cannot draw general conclusions about the GC systems of ellipticals and spirals, we can use the results for NGC 4472 to test a few specific predictions of models for galaxy formation.

Here we consider four different galaxy formation models. The first is the monolithic collapse scenario (e.g., Larson 1975, Carlberg 1984, Arimoto \& Yoshii 1987), in which elliptical formation is modeled as the collapse of an isolated massive gas cloud, or "protogalaxy" at high redshift. Such scenarios would produce a GC system with a color distribution that has a smooth shape and single peak directly related to the metallicity of the population. A second type of scenario is that in which ellipticals are formed by mergers, e.g., by the merger of two or more spirals (e.g., Toomre 1977, Ashman \& Zepf 1992, Zepf \& Ashman 1993). The simple merger scenario suggested by Ashman \& Zepf (1992; hereafter AZ92) involves the merger of disk galaxies, and yields two

GC populations: a younger, spatially-concentrated, metal-rich system of clusters formed during the merger, and a spatially-extended, metal-poor system that comes from the halos of the disk galaxies. A model by Forbes, Brodie, \& Grillmair (1997; hereafter FBG97) combines elements of both collapse and merger-type scenarios, since it involves dissipational collapse as well as accretion of surrounding galaxies. In the FBG97 scenario, ellipticals form their GCs in distinct star formation phases. The first phase forms a system of metal-poor GCs, and the second phase (which occurs after the gas in the galaxy has been self-enriched) gives rise to a population of more metal-rich GCs. FBG97 suggest that the outer regions of giant cluster ellipticals contain a population of metal-poor GCs that have been captured either through tidal stripping or accretion 
of smaller galaxies. The most recent proposed formation model that makes predictions for GC system properties is that put forth by Côté, Marzke, \& West (1998; hereafter CMW98). CMW98 propose that giant ellipticals form by dissipational collapse, during which a metal-rich population of GCs is formed. The population of bluer, metal-poor GCs are subsequently captured from other galaxies through mergers or tidal stripping.

Radial profile and local specific frequency. Because our fractional areal coverage drops beyond $\sim 17^{\prime}$ from the center of NGC 4472 , we can by no means state unequivocally that we have observed the entire extent of the GC system. Our observed radial profile does indicate, however, that it is unlikely that large numbers of GCs reside at radial distances beyond $\sim 23$. Models such as FBG97 and CMW98, that invoke tidal stripping as the mechanism by which GC systems of giant cluster ellipticals are formed, require that a large reservoir of GCs be present in the outer regions of galaxies. We have observed NGC 4472 out to $\sim 110 \mathrm{kpc}$ and do not find significant numbers of GCs at those radial distances. Indeed, the local specific frequency of GCs remains below 15 out to $23^{\prime}$.

Ratio of red to blue clusters. Like previous authors (e.g., Geisler et al. 1996, Puzia et al. 1999), we find that NGC 4472's GC system has a bimodal color distribution. A simple monolithic collapse model cannot explain the presence of two GC populations, so it appears that this particular galaxy, at least, has a more complicated formation history. We also find, as did Geisler et al. (1996), that the ratio of red (metal-rich) to blue (metal-poor) clusters in NGC 4472 is less than one. The AZ92 model, which attempted to explain why elliptical galaxies have specific frequencies $\gtrsim 2$ times those of spirals, suggests that the merger of two or more spiral galaxies creates a population of GCs comparable in size to the number originally contained in the spirals. This has the result of increasing $S_{N}$ by the required amount, and requires that $N_{R G C} / N_{B G C} \gtrsim 1$. This is not observed in NGC 4472, in disagreement with the AZ92 model. On the other hand, the total number of GCs in ellipticals compared to spirals is still not well-known. It is possible that the differences between the specific frequencies of typical ellipticals and spirals are smaller than previously thought, in which case the requirement from AZ92 that an equal number of GC's be created in the merger would no longer hold. These issues are explored further in the discussion below about global specific frequency.

Color gradient. All of the models we are considering can explain why the blue population of GCs in ellipticals would be more spatially extended than the red GCs. Lee et al. (1998) observed a color gradient in the total GC population of NGC 4472 out to $\sim 7^{\prime}$ and showed that this was caused by the increasing ratio of blue to red GCs at large radius. We observe a similar (although smaller) color gradient in the inner portion of the galaxy, but the gradient vanishes for the total population over the entire radial extent of the data. The flattening of the color gradient is not predicted by, nor consistent with, any of the proposed models. In fact, if the zero-gradient result is real, it raises some interesting questions about how the metal-rich GCs were formed at large radii.

Global specific frequency. We find a smaller global specific frequency for NGC 4472 than previous 
studies, although our value agrees with some previous values within the errors. We maintain that our GC sample is significantly less contaminated compared to past studies, due to our combined techniques of observing through multiple filters and using image analysis to eliminate extended objects. Moreover, using spectroscopic data and HST images, we have accurately quantified the amount of contamination in the sample so that we can then correct for it. Consequently we are better able to constrain the total number of GCs in NGC 4472.

Accurately establishing the total numbers of GCs in both spirals and ellipticals is of central importance in the study of extragalactic GC systems. Although ellipticals are generally thought to have specific frequencies at least twice as large as those for spirals, the quantitative difference between spirals and ellipticals is still uncertain. The reasons for this are that few spirals have been studied in detail, and the uncertainties in individual measurements of specific frequency for both spirals and ellipticals are large. Past studies have typically used photographic plates or small-area CCD's, and thus had low sensitivity and/or poor spatial coverage, and also had higher levels of contamination. Accurately-determined specific frequencies provide an important test of formation models, since all the models seek to explain the differences in specific frequencies for disk galaxies versus ellipticals.

One of the main goals of our overall study is to better determine the total numbers of GCs for each of a large sample of spiral galaxies, in order to test the prediction made by AZ92 that the mass-normalized number of metal-poor GCs around ellipticals and spirals should be the same. Even with the somewhat smaller total number of GCs we have observed in NGC 4472, it appears that there may be too many blue GCs in this galaxy to have originated in the halos of typical spirals. A useful parameter for comparing the total numbers of GCs in galaxies of widely varying masses is the $T$ parameter, the number of GCs per stellar galaxy mass (Zepf \& Ashman 1993). $T$ is defined as:

$$
T \equiv \frac{N_{G C}}{M_{G} / 10^{9} \mathrm{M}_{\odot}}
$$

where $M_{G}$ is the mass of the host galaxy. The total number of clusters in NGC 4472 out to $23^{\prime}$ is $\sim 5,900$; using this and the ratio of red to blue GCs, we calculate that about 3,500 of the GCs are blue and metal-poor. In the AZ92 picture, these are the clusters that originated in the halos of the progenitor spirals that merged to form NGC 4472. Assuming a mass-to-light ratio $M / L_{V}=10$ for ellipticals (cf. Faber \& Gallagher 1979), we obtain $T=2.6$ for the metal-poor GCs in NGC 4472.

If we calculate $T$ for the entire GC system of the two most well-studied spirals, the Milky Way and M31, we obtain $T$ values of approximately 1 and 2, respectively. (Here we have used $N_{G C}=$ 180 and $M / L_{V}=5$ for the Galaxy, and $N_{G C}=450$ and $M / L_{V}=6$ for M31.) If we assume that the Milky Way and M31 are typical spirals, then it would appear that even with our scaled-down total number of GCs, the AZ92 scenario cannot account for the blue cluster population observed in giant ellipticals like NGC 4472.

The assumption that the Milky Way and M31 are typical may not be a valid one. There are a few spiral galaxies that have been found by previous studies to have $N_{G C} \sim 900-1,000$ (see 
summary in Appendix of Ashman \& Zepf (1998)), yielding $T$ values $\gtrsim 3$. However, in some of these cases less than $10 \%$ of the estimated number of GCs were actually observed, making the $T$ values highly uncertain. Still other spirals have been found to have $T<1$, and the total number of GCs around a "typical" spiral is very much an open question. Based on our experience with NGC 4472, it is possible that when we analyze the data for the spiral galaxies in our sample, we will (because of our techniques for reducing contamination) find smaller specific frequencies for them as well.

In conclusion, our observations are consistent with some aspects of each of the models considered, and inconsistent with at least one prediction or assumption of each model. AZ92 make specific predictions concerning the characteristics of the GC systems of individual galaxies, so their model was perhaps the simplest to test using only one galaxy from our larger sample. It seems likely from this and other studies of NGC 4472 that the gaseous merger scenario as proposed by AZ92 is perhaps overly simplistic and that more complicated formation processes must be invoked in order to explain exactly how the GC system of NGC 4472 originated. Our study of isolated spirals and ellipticals in various environments is designed to help answer some of the outstanding questions concerning extragalactic GC systems, and address some of the more detailed predictions

of FBG97 and CMW98, both of which make testable predictions concerning the characteristics of the GC systems of ellipticals in general.

\section{Summary}

We have recently begun a study of the GC systems of a large sample of elliptical and spiral galaxies, in order to test the predictions of models for elliptical galaxy formation. Below we summarize our findings for the first elliptical observed for this study, the giant Virgo galaxy NGC 4472:

1. A de Vaucouleurs law of the form $\log \sigma_{G C}=(3.38 \pm 0.08)-(1.56 \pm 0.05) r^{1 / 4}$ provides a fairly good fit to the radial profile of NGC 4472's GC system, out to $\sim 16-17^{\prime}$. At larger radii, the observed profile falls slightly below the de Vaucouleurs law, and the number density of GCs is consistent with zero in the region from $21^{\prime}$ to $23^{\prime}$.

2. The bimodal color distribution in NGC 4472's GC system observed by previous authors is apparent in our $B-R$ distribution. The overall ratio of red to blue GCs is $\sim 0.7$.

3. For GCs located within $8^{\prime}$ of the galaxy center, we find a small negative color gradient: the GC system becomes bluer with increasing radius, due to the increasing ratio of blue to red clusters. The gradient disappears, however, when the entire GC population out to $22^{\prime}$ is taken into account; we find no color gradient in the total GC population.

4. The local specific frequency rises approximately linearly with radius out to $\sim 10^{\prime}(\sim 50 \mathrm{kpc})$. At larger radii, the local $S_{N}$ values scatter substantially above and below the values predicted by the de Vaucouleurs law fit to the radial profile. In the region from $17-23^{\prime}$, the observed local 
$S_{N}$ appears to turn over and begin to decrease. Our values of local $S_{N}$ are significantly lower than those found by Lee et al. (1998) using ground-based data, but agree fairly well with values calculated from an HST study by Puzia et al. (1999). We do not find, as suggested by McLaughlin (1999), that local $S_{N}$ is constant at all radii beyond $\sim 15 \mathrm{kpc}$.

5. There are $\sim 5,900$ clusters in NGC 4472's GC system out to $23^{\prime}$, yielding a global specific frequency within that radius of $3.6 \pm 0.6$.

6. We have compared our results with the predictions of four different galaxy formation models, and find that the results are inconsistent with at least one aspect of each model. Our observed radial profile indicates it is unlikely that a large population of GCs resides at radii beyond $\sim 23^{\prime}$, challenging formation models that require a substantial reservoir of GCs in the far outer regions of galaxies. The ratio of red to blue clusters for NGC 4472 is at odds with a prediction of the simple merger scenario that the numbers of metal-rich and metal-poor GCs should be comparable. The lack of a color gradient in the total GC population is not explained nor predicted by any of the models considered. Finally, although our calculated total number of GCs in NGC 4472 is smaller than previous estimates, it appears that there are still slightly more metal-poor GCs per unit mass in NGC 4472 than can be accounted for by merging the halo GC populations of spiral galaxies like the Milky Way and M31.

K.L.R. gratefully acknowledges financial support from a NASA Graduate Student Researchers Fellowship during the preparation of this paper. S.E.Z. acknowledges support from the Hellman Family Fellowship and NASA Long-Term Space Astrophysics grant NAG5-9651. We are grateful to Thomas Puzia for providing an electronic version of the GC candidate list from Puzia et al. (1999), and to Charles Liu for providing electronic versions of the template galaxy spectra used for the color-color plane analysis. We thank the staff at Kitt Peak National Observatory for their assistance during our 4-meter observing run. We also thank John Salzer, Doug Geisler, Thomas Puzia, and the anonymous referee for useful comments that have improved the quality of the paper. This research has made use of the NASA/IPAC Extragalactic Database (NED), which is operated by the Jet Propulsion Laboratory, California Institute of Technology, under contract with the National Aeronautics and Space Administration. 


\section{REFERENCES}

Arimoto, N., \& Yoshii, Y. 1987, A\&A, 173, 23

Ashman, K.M., Bird, C.M., \& Zepf, S.E. 1994, AJ, 108, 2348

Ashman, K.M., \& Zepf, S.E. 1992, ApJ, 384, 50

Ashman, K.M., \& Zepf, S.E. 1998, Globular Cluster Systems (Cambridge: Cambridge University Press)

Bahcall, J.N., \& Soneira, R.M. 1980, ApJS, 44, 73

Caon, N., Capaccioli, M., \& D’Onofrio, M. 1994, A\&AS, 106, 199

Carlberg, R.G. 1984, ApJ, 286, 403

Coleman, G.D., Wu, C.-C., \& Weedman, D.W. 1980, ApJS, 43, 393

Côté, P., Marzke, R.O., \& West, M.J. 1998, ApJ, 501, 554

de Vaucouleurs, G., de Vaucouleurs, A., Corwin Jr., H.G., Buta, R.J., Paturel, G., \& Fouque, P. 1991, Third Reference Catalogue of Bright Galaxies (New York: Springer)

Faber, S.M., \& Gallagher, J.S. 1979, ARA\&A, 17, 135

Forbes, D.A., Brodie, J.P., \& Grillmair, C.J. 1997, AJ, 113, 1652

Gebhardt, K., \& Kissler-Patig, M. 1999, AJ, 118, 1526

Geisler, D. 1996, AJ, 111, 480

Geisler, D., Lee, M.G., \& Kim, E. 1996, AJ, 111, 1529

Harris, W.E. 1986, AJ, 91, 822

Harris, W.E. 1991, ARA\&A, 29, 543

Harris, W.E. 1996, AJ, 112, 1487

Harris, W.E. \& van den Bergh, S. 1981, AJ, 86, 1627

Kennicutt, R.C. Jr. 1992, ApJS, 79, 255

Kim, E., Lee, M.G., \& Geisler, D. 2000, MNRAS, in press

King, I.R. 1978, ApJ, 222, 1

Kinney, A.L., Calzetti, D., Bohlin, R.C., McQuade, K., Storchi-Bergmann, T., \& Schmitt, H.R. 1996, ApJ, 467, 38 
Kundu, A., \& Whitmore, B.C. 1999, BAAS, 194, 35.04

Kundu, A., Whitmore, B.C., Sparks, W.B., \& Macchetto, F.D. 1999, ApJ, 513, 733

Landolt, A.U. 1992, AJ, 104, 1

Larson, R.B. 1975, MNRAS, 173, 671

Lee, M.G., \& Kim, E. 2000, AJ, 120, 260

Lee, M.G., Kim, E., \& Geisler, D. 1998, AJ, 115, 947

McLaughlin, D.E. 1999, AJ, 117,2398

Neilsen, E.H., Jr. 2000, PASP, 112, 120

Puzia, T.H., Kissler-Patig, M., Brodie, J.P., \& Huchra, J.P. 1999, AJ, 118, 2734

Rhode, K.L., \& Zepf, S.E. 2001, in preparation

Schlegel, D.J., Finkbeiner, D.P., \& Davis, M. 1998, ApJ, 500, 525

Sharples, R.M., Zepf, S.E., Bridges, T.J., Hanes, D.A., Carter, D., Ashman, K.M., \& Geisler, D. 1998, AJ, 115, 2337

Toomre, A. 1977, in The Evolution of Galaxies and Stellar Populations, eds. B. Tinsley \& R. Larson (Yale Univ. Obs., New Haven)

Whitmore, B.C., Sparks, W.B., Lucas, R.A., Macchetto, F.D., \& Biretta, J.A. 1995, ApJ, 454, L73

Zepf, S.E., \& Ashman, K.M. 1993, MNRAS, 264, 611

Zepf, S.E., Beasley, M.A., Bridges, T.J., Hanes, D.A., Sharples, R.M., Ashman, K.M., \& Geisler, D. 2000, AJ, in press 

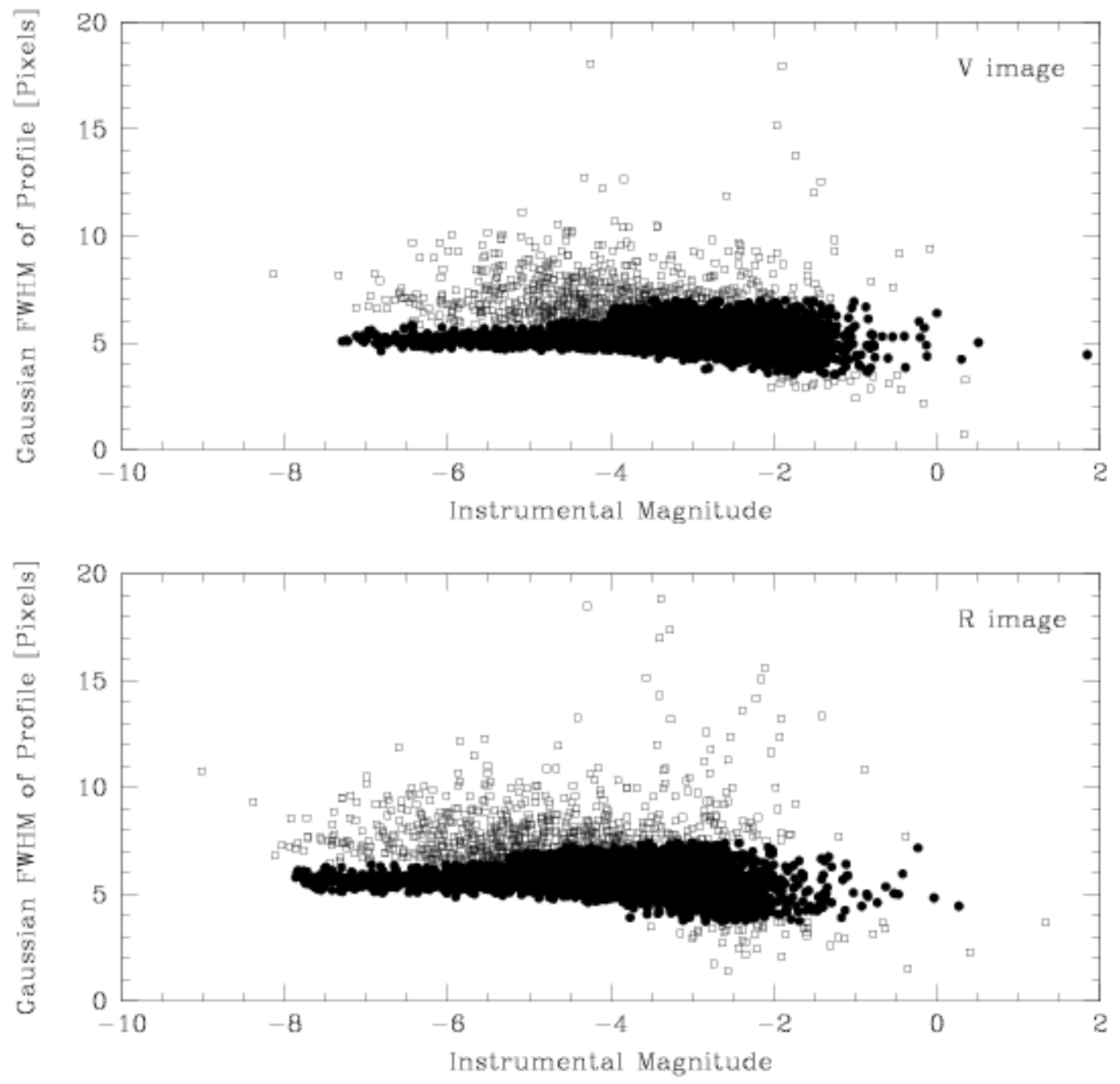

Fig. 1.- Extended source cut. Gaussian FWHM of the radial profile versus instrumental magnitude for the 4,329 sources in the initial GC candidate list in the $V$ - and $R$-band images. Filled circles are objects that pass the FWHM cut; open squares are objects eliminated from the GC candidate list at this step. 


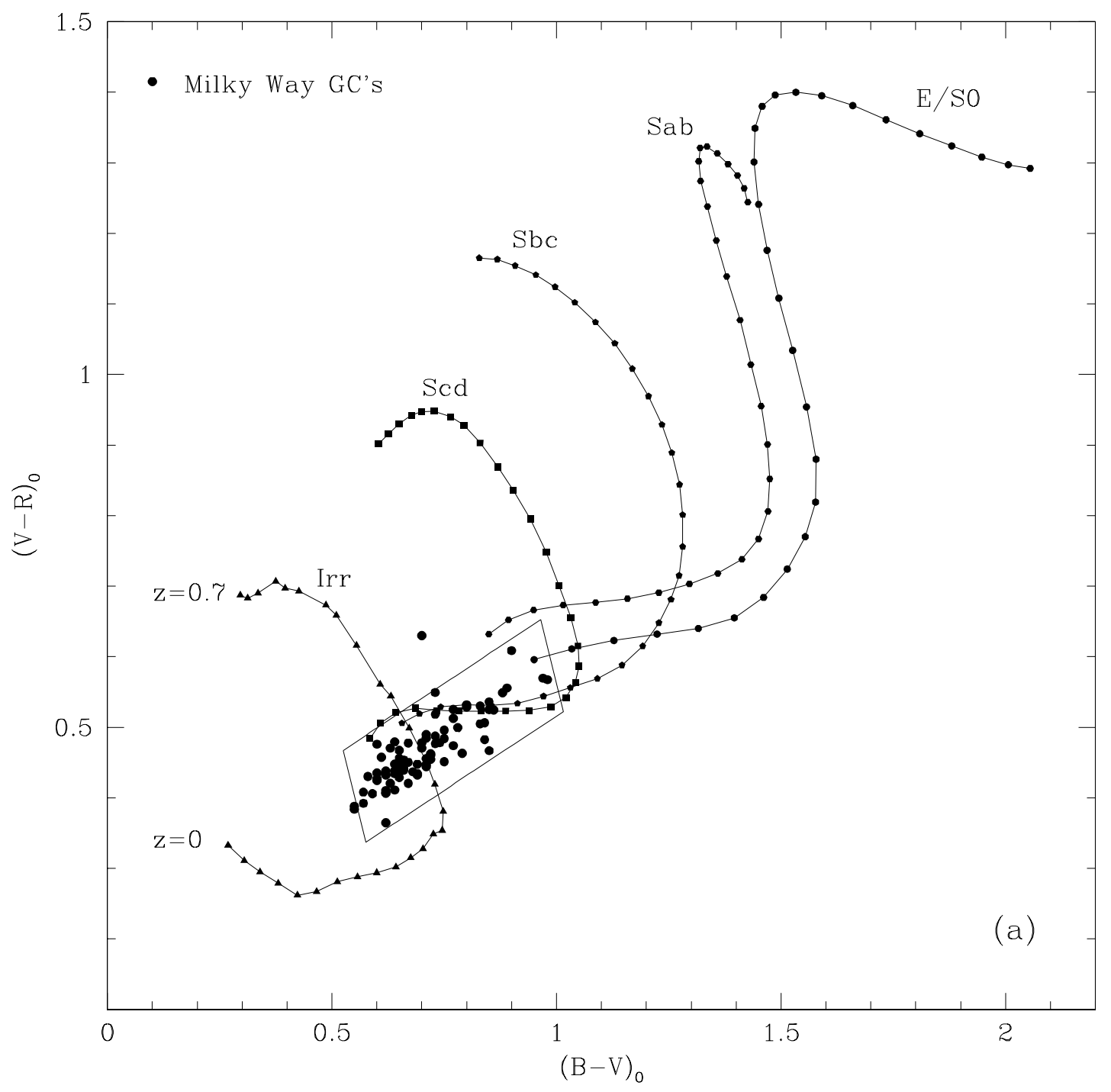

Fig. 2.- (a) Milky Way GCs in the BVR color-color plane. The rectangle marks the region where GCs would be expected to appear in this color-color plane. Milky Way GCs from the Harris catalog (Harris 1996) are shown as filled circles. The positions of galaxies of different morphological types appear as "tracks" the galaxies would follow with increasing redshift (see Section 3.4 for details). (b) Color selection. Open squares mark the positions of the 3,458 GC candidates that passed the extended source cut in the $B V R$ color-color plane. Filled circles mark the 1,465 sources whose colors and associated photometric errors put them within 2-sigma of the $V-R$ versus $B-V$ relation for GCs. For reference, the galaxy tracks that appear in Figure $2 a$ are also shown here. The reddening vector appears in the upper left corner, and its length corresponds to $A_{V}=1$ magnitude. 


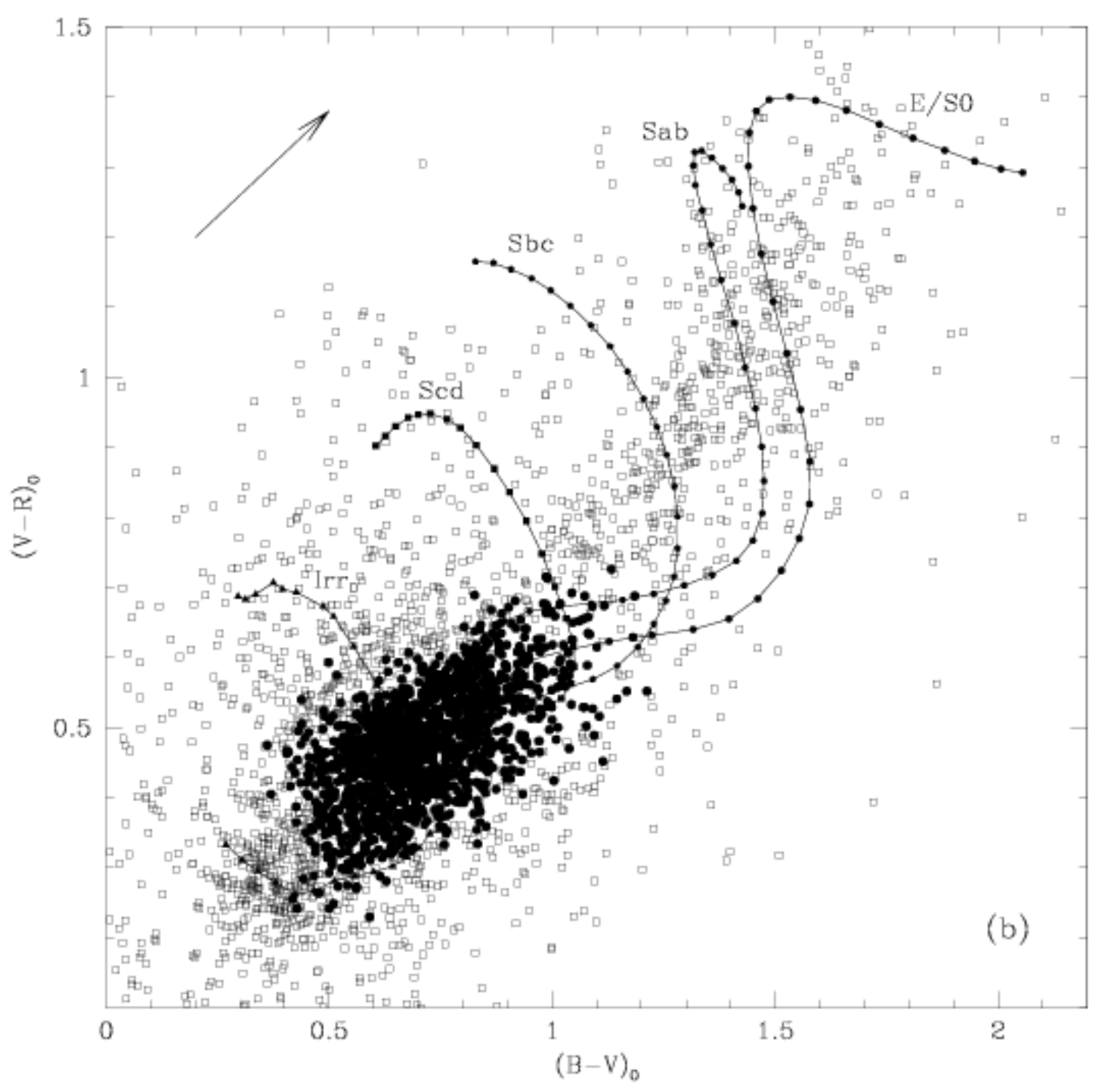




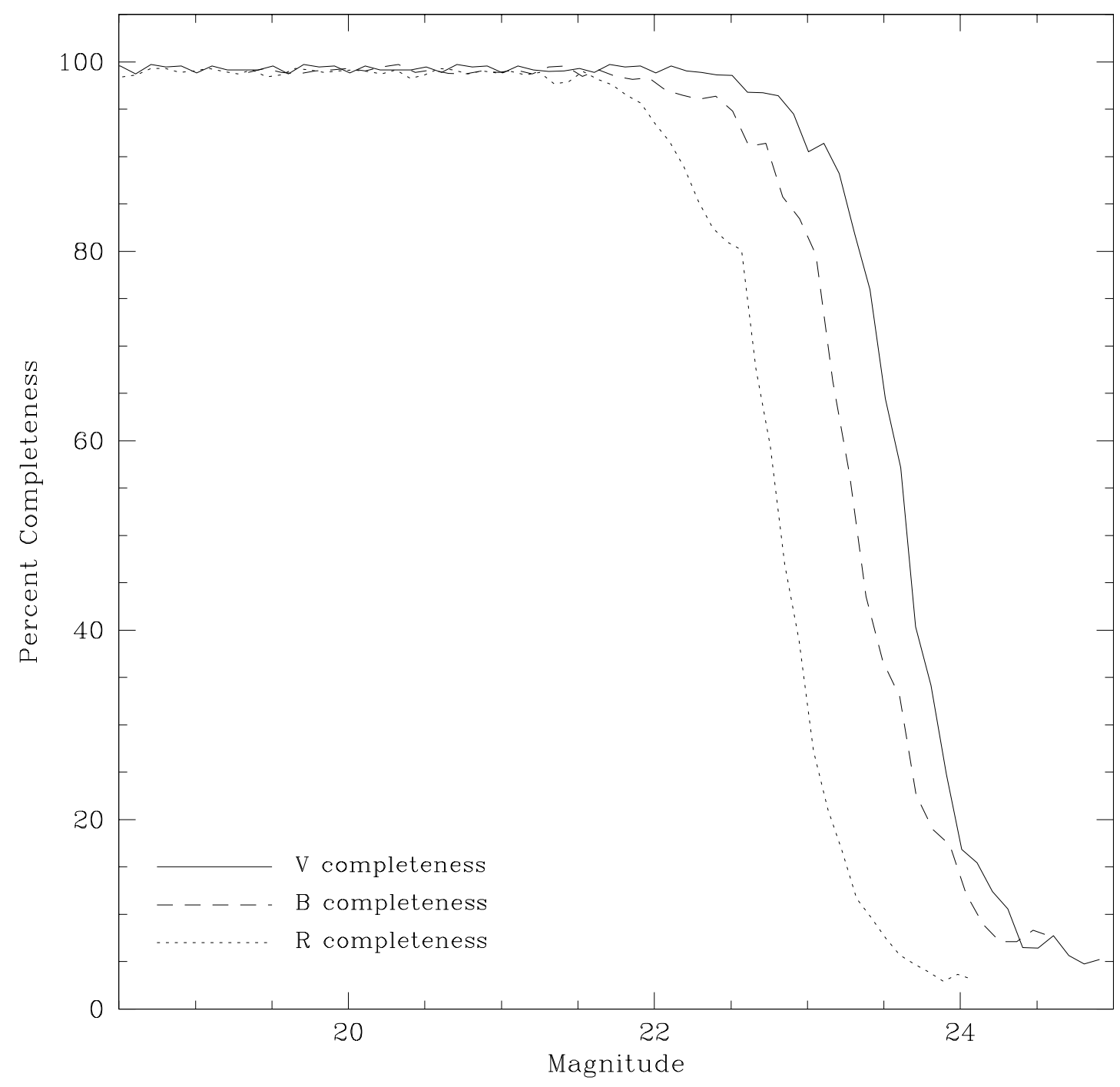

Fig. 3.- Completeness as a function of magnitude. The detection limits for GCs for our images were determined using artificial star tests, as described in Section 3.5. The resultant completeness curves for each filter are shown here. 


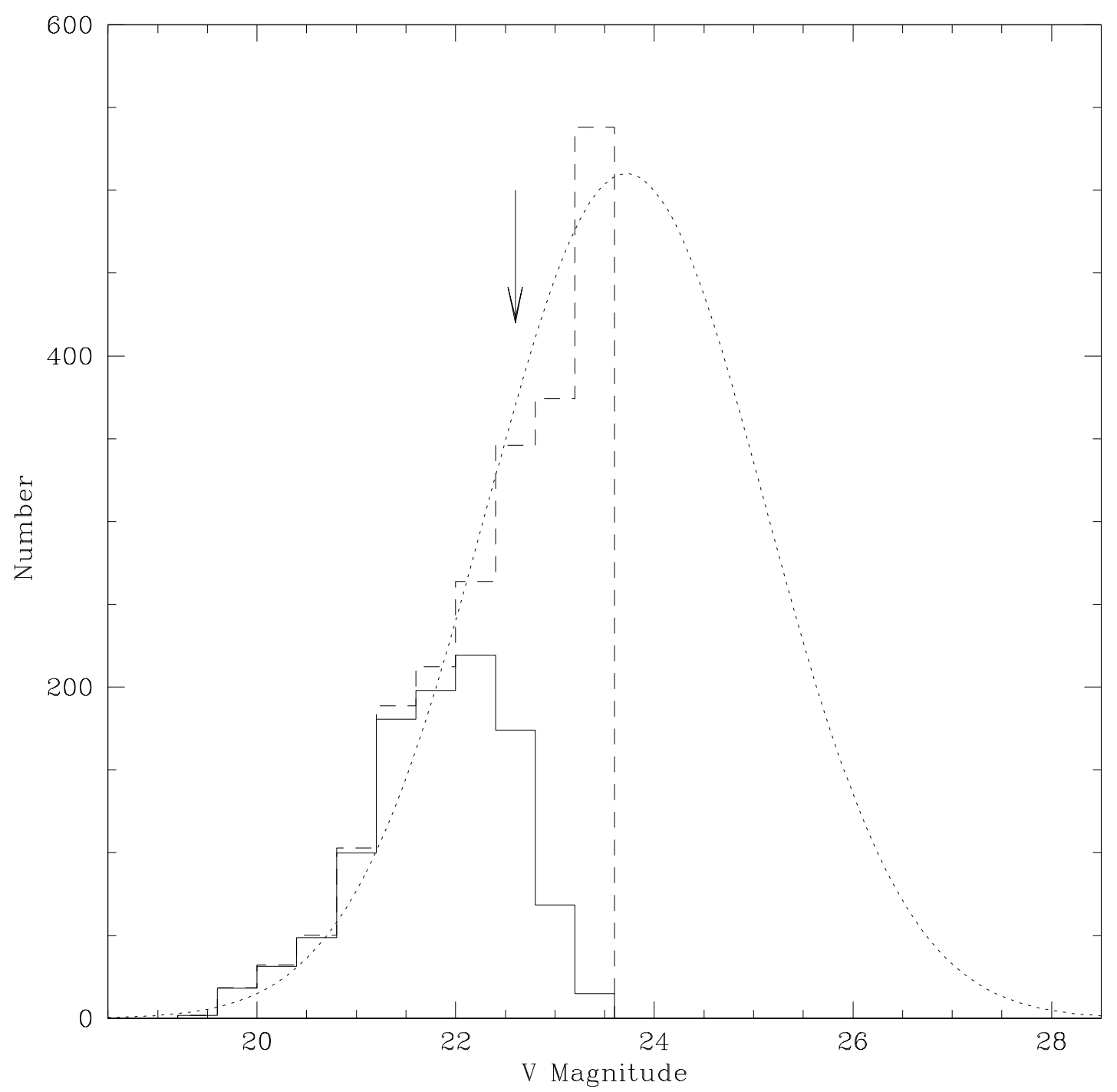

Fig. 4.- Globular Cluster Luminosity Function. The solid line marks the observed $V$-band luminosity function for $1,279 \mathrm{GC}$ candidates with $V \leq 23.5$, located within $16^{\prime}$ of the center of NGC 4472. The dashed line shows the same GCLF corrected for incompleteness in the filter combination $(B, V$, and $R)$ used to select the GC candidates. The arrow points to the bin where the combined completeness is 50\%. The M87 GCLF from Whitmore et al. (1995), normalized to our data, is shown as a dotted line. 

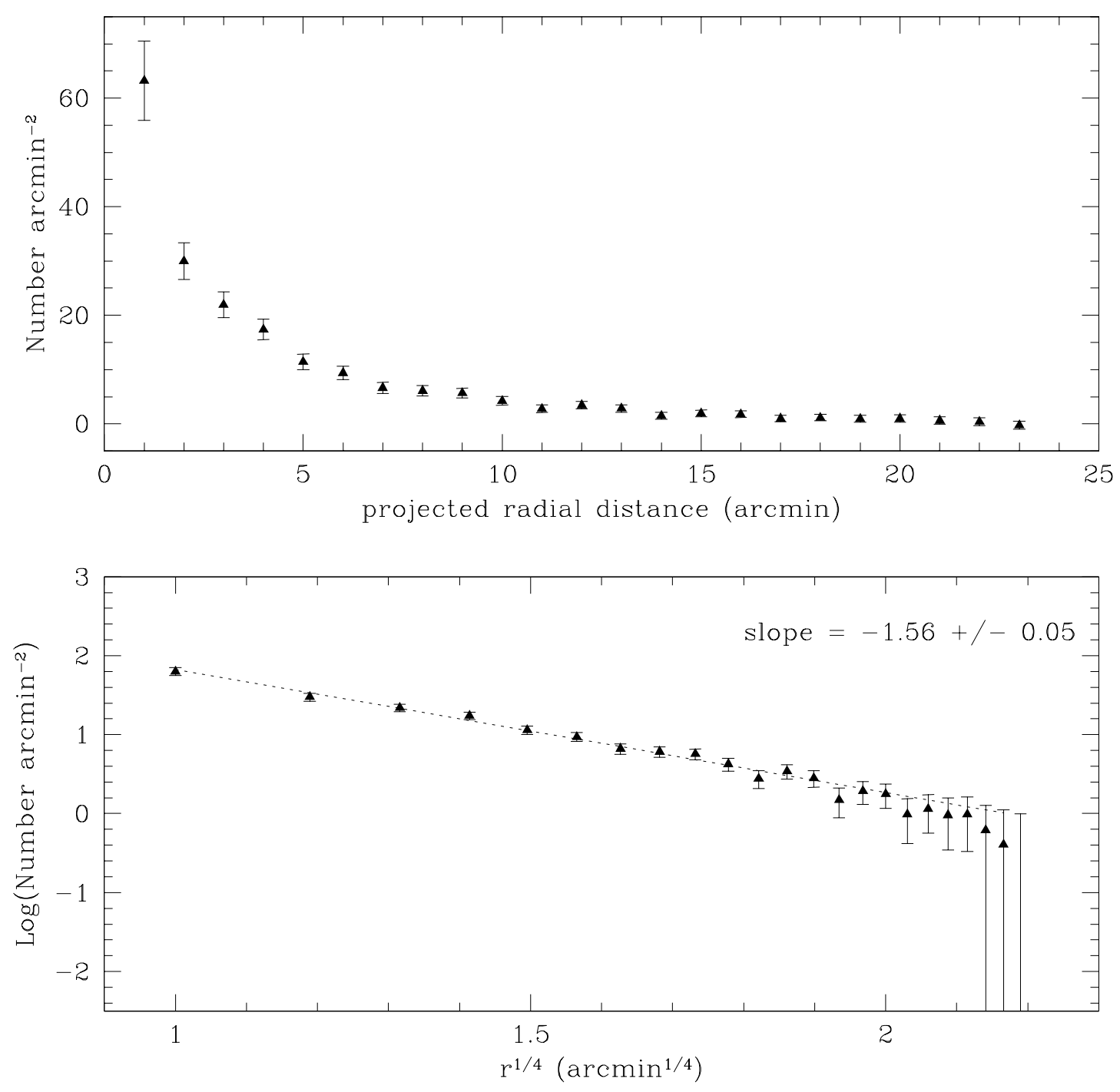

Fig. 5.- Radial distribution of GCs. The upper plot shows the surface density of GCs in NGC 4472 as a function of projected radial distance. The lower plot shows the log of the surface density versus $\mathrm{r}^{1 / 4}$, and the dotted line is the best-fit de Vaucouleurs profile. The profiles have been corrected for contamination, areal coverage, and magnitude incompleteness, as described in Section 4.1. 


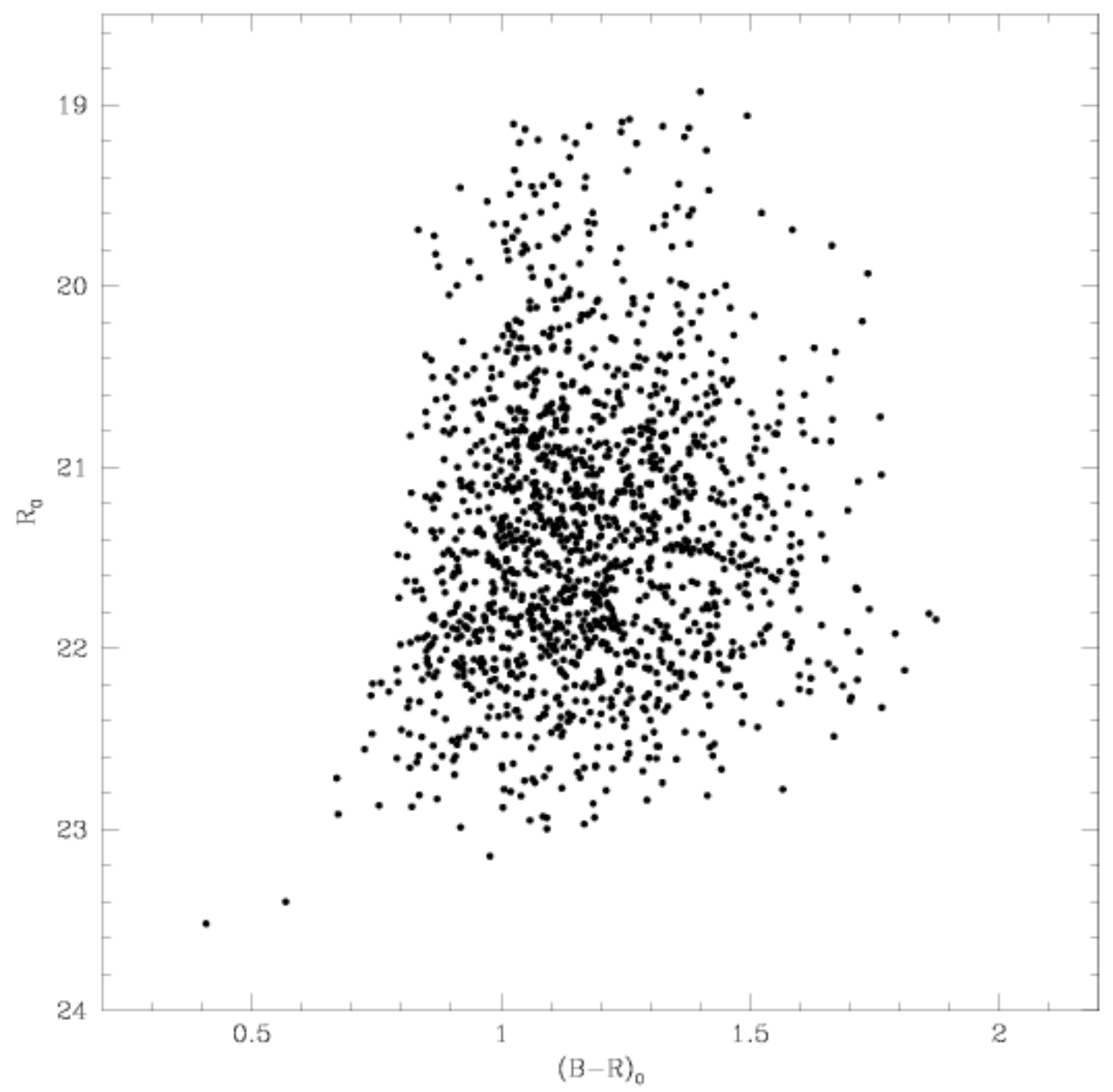

Fig. 6.- Color-magnitude diagram for GC candidates. $R$ magnitudes for 1,465 GC candidates are shown here versus their $B-R$ colors. The relative shallowness of the $B$ image means that we are more sensitive to detecting blue GCs than red ones. 


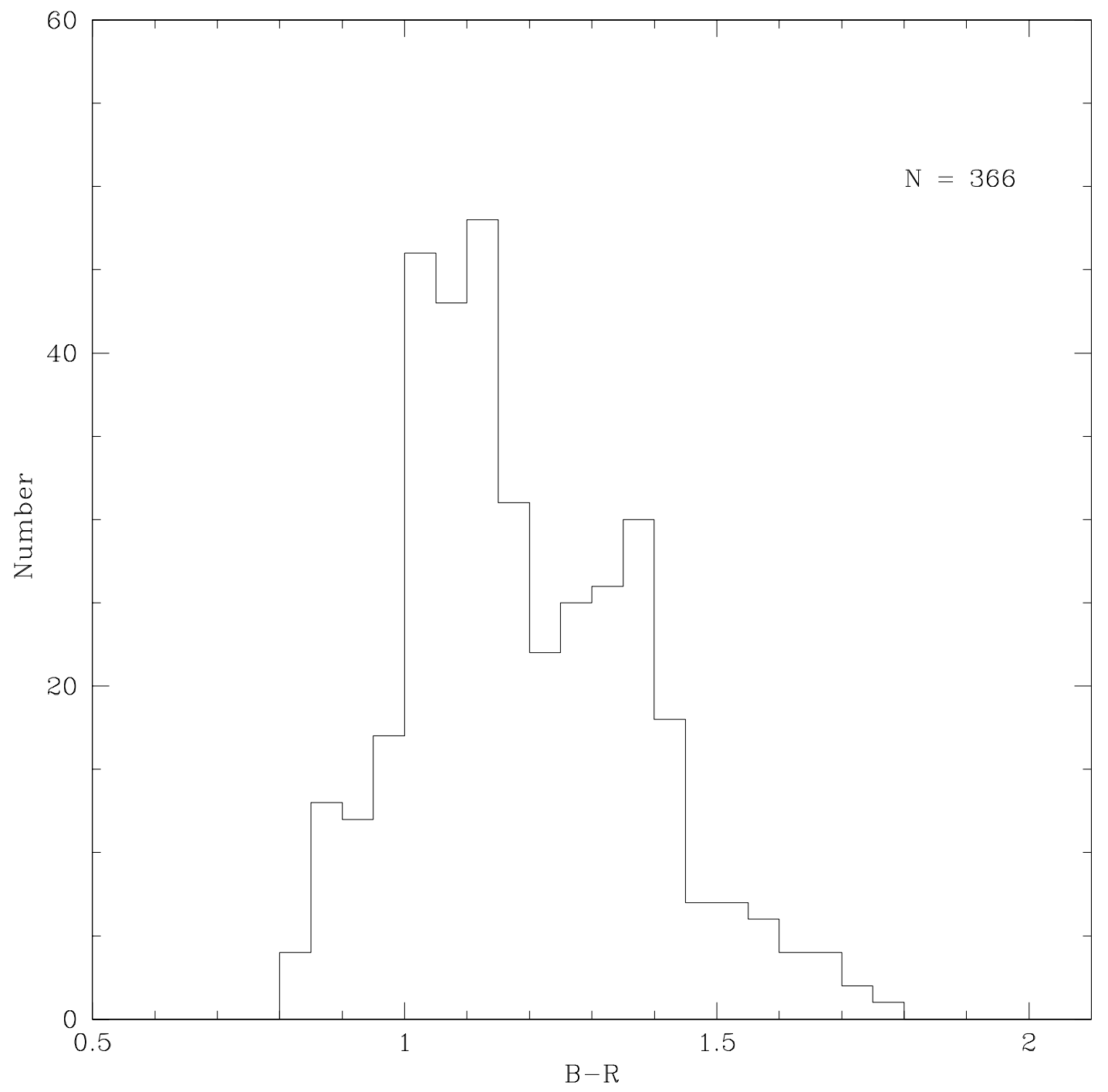

Fig. 7.- Color distribution of GC candidates. This plot shows the color distribution of a sub-sample of objects taken from the total sample of 1,465 GC candidates and selected to be $90 \%$ complete for the blue population and complete for the red population. The $B-R$ distribution is bimodal, with peaks around $B-R=1.10$ and 1.35 , and a gap at 1.23. 

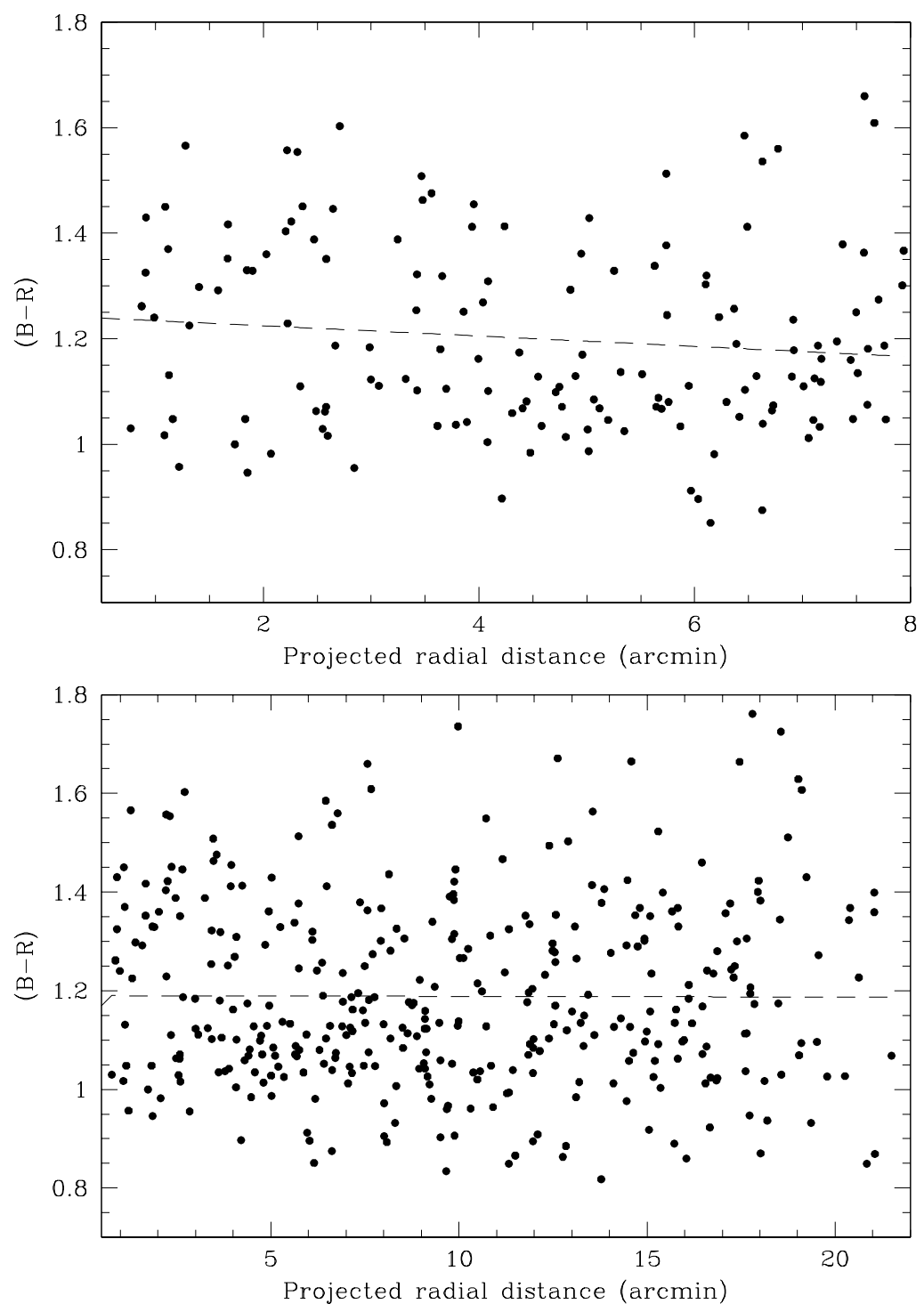

Fig. 8.- Color versus projected radial distance for 366 GC candidates. These plots show $B-R$ color versus radius for the $90 \%$ sample of GC candidates, for the inner $8^{\prime}$ of NGC 4472 (upper plot) and the entire radial region covered by our data (lower plot). The small gradient seen in the color distribution for the inner region is due to a slight overdensity of blue clusters in the radial range $4^{\prime}$ to $8^{\prime}$. The color gradient vanishes in the total population for the full radial range of the data. Dashed lines are best-fit lines to the data shown. 


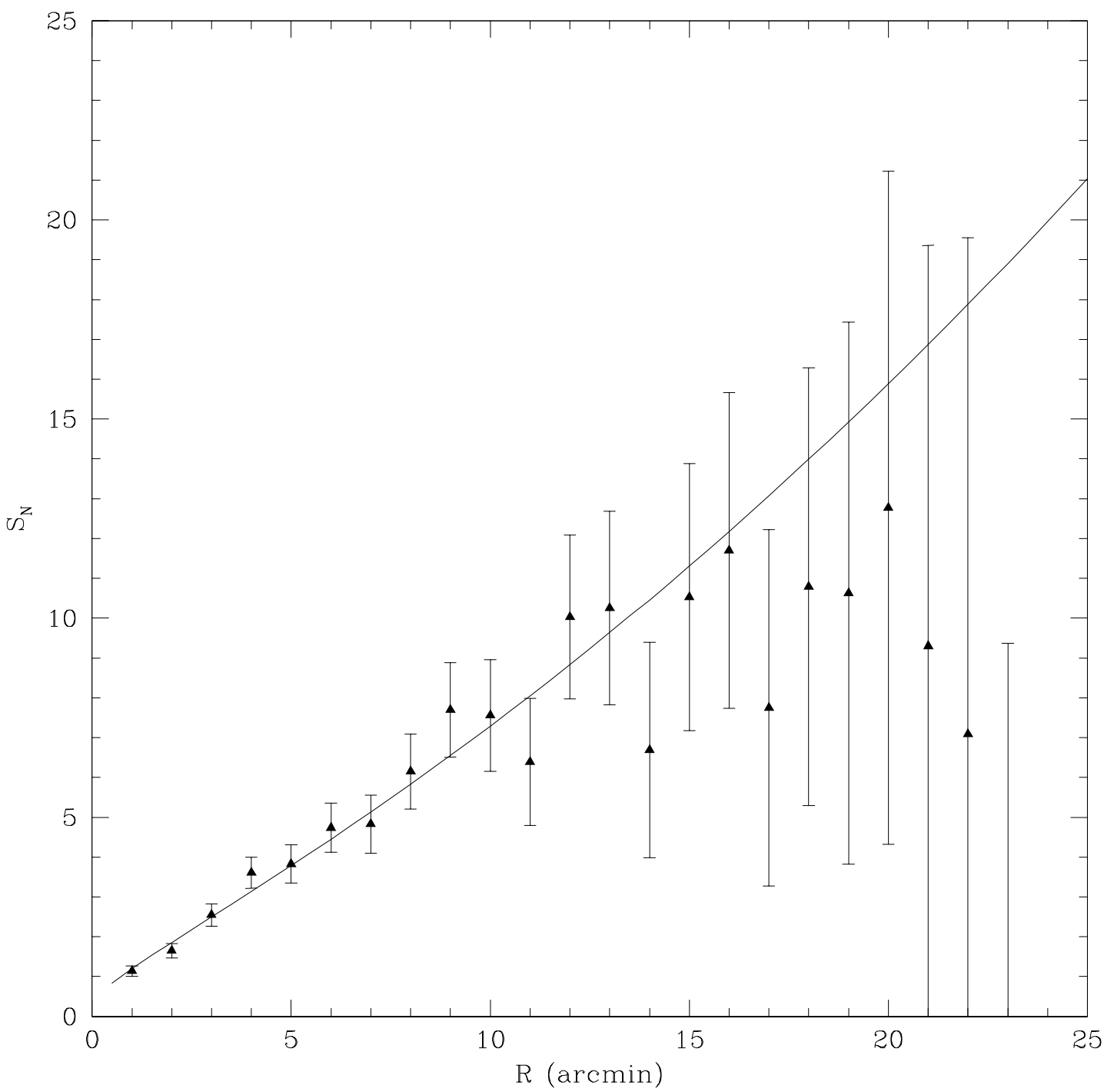

Fig. 9.- Local specific frequency of GCs. This figure shows how $S_{N}$ varies with radius in NGC 4472. Filled triangles are points calculated using our GC sample and surface brightness profile for NGC 4472. The solid line shows the expected $S_{N}$ based on the best-fit de Vaucouleurs law fit to the radial distribution of GCs. The errors in $S_{N}$ become larger as fewer GCs are detected within the area of each annular region. 
Table 1. Aperture Corrections Used for Photometry of GC Candidates

\begin{tabular}{lc}
\hline \hline Image & Aperture Correction \\
\hline$B$ & $-0.2112 \pm 0.0184$ \\
$V$ & $-0.1970 \pm 0.0117$ \\
$R$ & $-0.1376 \pm 0.0176$ \\
\hline
\end{tabular}

Table 2. Corrected Radial Profile for GCs in NGC 4472

\begin{tabular}{crc}
\hline \hline $\begin{array}{c}\text { Radius } \\
(\operatorname{arcmin})\end{array}$ & \multicolumn{1}{c}{$\begin{array}{c}\sigma \\
\left(\operatorname{arcmin}^{-2}\right)\end{array}$} & Fractional coverage \\
\hline 1 & $63.23 \pm 7.33$ & 0.86 \\
2 & $29.98 \pm 3.37$ & 1.00 \\
3 & $21.93 \pm 2.40$ & 1.00 \\
4 & $17.39 \pm 1.88$ & 1.00 \\
5 & $11.44 \pm 1.42$ & 1.00 \\
6 & $9.38 \pm 1.22$ & 1.00 \\
7 & $6.64 \pm 1.01$ & 1.00 \\
8 & $6.10 \pm 0.94$ & 1.00 \\
9 & $5.68 \pm 0.88$ & 1.00 \\
10 & $4.24 \pm 0.78$ & 1.00 \\
11 & $2.79 \pm 0.70$ & 0.98 \\
12 & $3.45 \pm 0.71$ & 1.00 \\
13 & $2.82 \pm 0.67$ & 1.00 \\
14 & $1.49 \pm 0.60$ & 1.00 \\
15 & $1.93 \pm 0.61$ & 1.00 \\
16 & $1.77 \pm 0.60$ & 1.00 \\
17 & $0.98 \pm 0.57$ & 1.00 \\
18 & $1.15 \pm 0.59$ & 0.81 \\
19 & $0.96 \pm 0.61$ & 0.54 \\
20 & $0.98 \pm 0.65$ & 0.39 \\
21 & $0.61 \pm 0.66$ & 0.28 \\
22 & $0.40 \pm 0.71$ & 0.18 \\
23 & $-0.25 \pm 0.71^{\dagger}$ & 0.10 \\
\hline
\end{tabular}

${ }^{\dagger}$ A subtractive correction for contamination has been applied to the surface density of GCs in each bin, resulting in a negative surface density for the outermost bin. 
Table 3. Radial Variation of Specific Frequency for GCs in NGC 4472

\begin{tabular}{cr}
\hline \hline Radius (arcmin) & \multicolumn{1}{c}{$S_{N}$} \\
\hline 1 & $1.14 \pm 0.13$ \\
2 & $1.65 \pm 0.18$ \\
3 & $2.55 \pm 0.28$ \\
4 & $3.61 \pm 0.39$ \\
5 & $3.83 \pm 0.48$ \\
6 & $4.74 \pm 0.62$ \\
7 & $4.83 \pm 0.73$ \\
8 & $6.15 \pm 0.94$ \\
9 & $7.70 \pm 1.19$ \\
10 & $7.56 \pm 1.40$ \\
11 & $6.39 \pm 1.60$ \\
12 & $10.03 \pm 2.06$ \\
13 & $10.25 \pm 2.43$ \\
14 & $6.69 \pm 2.70$ \\
15 & $10.53 \pm 3.35$ \\
16 & $1.70 \pm 3.96$ \\
17 & $7.75 \pm 4.47$ \\
18 & $10.79 \pm 5.50$ \\
19 & $10.63 \pm 6.80$ \\
20 & $12.77 \pm 8.45$ \\
21 & $9.30 \pm 10.06$ \\
22 & $7.09 \pm 12.46$ \\
23 & $-5.06 \pm 14.43$ \\
\hline
\end{tabular}

